\title{
Evidence-based care in high- and low-risk groups following whiplash injury: a multi- centre inception cohort study
}

\author{
Alexandra Griffin ${ }^{1,2^{*}}$ (D), Jagnoor Jagnoor ${ }^{2,5}$, Mohit Arora ${ }^{2}$, lan D. Cameron ${ }^{2}$, Annette Kifley ${ }^{2}$, Michele Sterling ${ }^{3,4}$,
} Justin Kenardy ${ }^{4}$ and Trudy Rebbeck ${ }^{1,2}$

\begin{abstract}
Background: Studies aimed at improving the provision of evidence-based care (EBC) for the management of acute whiplash injuries have been largely successful. However, whether EBC is broadly provided and whether delivery of EBC varies based on risk of non-recovery, is uncertain. Receiving EBC should improve recovery, though this relationship has yet to be established. Further, mitigating the effect of EBC is the relationship with the practitioner, a phenomenon poorly understood in WAD. This study aimed to determine the proportion of individuals with whiplash, at differing baseline risk levels, receiving EBC. This study also aimed to determine whether receiving EBC and the therapeutic relationship were associated with recovery at 3 months post injury.
\end{abstract}

Methods: Participants with acute whiplash were recruited from public hospital emergency departments, private physiotherapy practices, and State Insurance Regulatory Authority (SIRA) databases. Participants completed questionnaires at baseline (demographics, risk of non-recovery) and 3-months (treatment received, risk identification, therapeutic relationship) post injury. Primary health care providers (HCPs) treating these participants also completed questionnaires at 3-months. Recovery was defined as neck disability index $\leq 4 / 50$ and global perceived effect of $\geq 4 / 5$.

Results: Two-hundred and twenty-eight people with acute whiplash, and 53 primary care practitioners were recruited. The majority of the cohort reported receiving EBC, with correct application of the Canadian C-spine rule (74\%), and provision of active treatments (e.g. 89\% receiving advice) high. Non-recommended (passive) treatments were also received by a large proportion of the cohort (e.g. $50 \%$ receiving massage). The therapeutic relationship was associated with higher odds of recovery, which was potentially clinically significant (OR 1.34, 95\% Cl 1.18-1.62). EBC was not significantly associated with recovery.

Conclusions: Guideline-based knowledge and practice has largely been retained from previous implementation strategies. However, recommendations for routine risk identification and tailored management, and reduction in the provision of passive treatment have not. The therapeutic relationship was identified as one of several important predictors of recovery, suggesting that clinicians must develop rapport and understanding with their patients to improve the likelihood of recovery.

Keywords: Whiplash injuries, Evidence-based health care, Clinical practice guidelines, Practice guidelines, Cohort

\footnotetext{
* Correspondence: agri3036@uni.sydney.edu.au

${ }^{1}$ Faculty of Health Sciences, The University of Sydney, 75 East St, Lidcombe,

NSW, Australia

2John Walsh Centre for Rehabilitation Research, The University of Sydney,

Kolling Institute, St Leonards, NSW, Australia

Full list of author information is available at the end of the article
}

C The Author(s). 2019 Open Access This article is distributed under the terms of the Creative Commons Attribution 4.0 International License (http://creativecommons.org/licenses/by/4.0/), which permits unrestricted use, distribution, and

reproduction in any medium, provided you give appropriate credit to the original author(s) and the source, provide a link to the Creative Commons license, and indicate if changes were made. The Creative Commons Public Domain Dedication waiver (http://creativecommons.org/publicdomain/zero/1.0/) applies to the data made available in this article, unless otherwise stated. 


\section{Background}

Recovery following whiplash injury, if it is to occur, will occur for the most part within the first 3 months post injury [1]. Current estimates suggest that approximately $50 \%$ of individuals will recover by 3 months post injury, whilst the remainder will experience mild to moderate long-term disability [1-3]. Since the majority of recovery in Australian cohorts occurs within the first 3 months following injury $[1,4]$, guidelines for the management of acute whiplash associated disorders (WAD) have an important role. In New South Wales (NSW), Australia, clinical practice guidelines for the management of acute WAD were developed with the purpose of improving management and patient outcomes. Recommendations were based on the National Health and Medical Research Council (NHMRC) grade of evidence [5]. Key recommendations were identified for implementation from these guidelines and included: (1) Appropriate imaging and selection of active treatments, (2) A reduction in the selection of passive treatments, and (3) An improvement in the process of care [6]. Whilst implementation of these recommendations in previous work [7-9] has improved the provision of appropriate care, little is known as to whether the provision of $\mathrm{EBC}$ and the process of care are maintained and associated with improved recovery. The translation of evidence to consumers, particularly patients and clinicians, via evidence-based, specific, and simple recommendations is essential for achieving compliance $[10,11]$.

The first key recommendation identified from the Australian whiplash guidelines was the appropriate application of the Canadian C-Spine rule. The Canadian $\mathrm{C}$-Spine rule is a widely validated rule used to assist clinicians in determining whether an $\mathrm{x}$-ray is required to confirm the diagnosis of a clinically important cervical injury [12]. The application of the rule involves working though a series of questions regarding risk factors for clinically important cervical spine injuries (i.e. cervical fracture or dislocation). Risk factors are weighted and presented in a flow-chart format. With correct application, the Canadian C-Spine rule is highly sensitive (range 0.90-1.00), and has the potential to reduce unnecessary exposure to radiation by as much as $42 \%$ [13]. However at present, the rate of unnecessary imaging in whiplash cohorts is unknown.

The provision of active treatments, such as advice and neck exercises, and the reduction of passive treatments with little or no evidence supporting their use, was also a key recommendation. The relative efficacy of active versus passive treatments is well established, [14-16] and whilst previous studies have demonstrated good uptake of the provision of active treatments, [7-9] to date it is unknown whether the provision of passive treatments has reduced.

The final key recommendation from the Australian whiplash guidelines was a differential process of management based on risk of non-recovery. Individuals at risk of nonrecovery should be identified early, using a recommended prognostic tool, to facilitate timely and appropriate multidisciplinary management. Recommended prognostic tools include measures of pain intensity using the visual analogue scale (VAS), or the numeric rating scale (NRS), and measures of neck pain-related disability using the neck disability index (NDI). Scores of $>5 / 10$ on the NRS or VAS, and $>$ $15 / 50$ on the NDI are associated with poorer recovery [6]. Additionally, a clinical prediction rule (CPR) to estimate risk of non-recovery in WAD has been developed [17] and validated [18]. This rule has a positive predictive value (PPV) of $71 \%$ in predicting individuals: (a) at risk of moderate to severe disability, and (b) likely to make full recovery, at 12 months post injury. The CPR incorporates age, the NDI and the hyperarousal sub scale of the post-traumatic diagnostic scale (PDS). Since its recent development, it is unknown whether clinicians are utilising this CPR, or any of the other prognostic indicators, to correctly identify individuals at risk of non-recovery, and tailor management accordingly.

Following early identification of risk, the guidelines recommend differential management. For example, it is recommended that individuals at low-risk of non-recovery have minimal care, and individuals at high risk be referred to WAD specialists, including rehabilitation physicians, specialist physiotherapists, psychologists and occupational physicians [6]. The provision of care based on risk has shown effectiveness in other musculoskeletal conditions, including low back pain [19]. It is proposed [20] yet unknown whether a similar risk stratification approach will result in improved health outcomes for whiplash. Examining current practice in regards to differential management strategies for WAD, based on risk, is an important starting point in this process.

Finally the relevance of the provision of EBC may be important only if it relates to recovery. Given recovery following whiplash injury is poor, it is important to establish the relationship between provision of EBC and recovery. Mitigating the effect of EBC is how it is delivered, namely the relationship with the practitioner. The therapeutic relationship refers to the sense of trust, warmth and support between patient and clinician, [21] and is positively associated with improved outcomes in low back pain [22] and mental health [23] populations. Whilst well established in psychotherapy research, the role of the therapeutic relationship in mediating outcomes in WAD is unknown. The therapeutic relationship, together with the provision of $\mathrm{EBC}$, may have a significant influence on outcomes following WAD, though this is currently unknown. Therefore, the aims of this study were to determine the proportion of people with WAD receiving $\mathrm{EBC}$, and whether baseline risk level, receiving $\mathrm{EBC}$ and the strength of the therapeutic relationship, were associated with recovery at 3 months post injury. 


\section{Methods}

\section{Study design}

This study was a prospective, multi-centre inception cohort study of patients with acute WAD.

\section{Participants}

Participants included individuals with whiplash [participants with whiplash], and their primary HCPs. Whiplash is defined, according to the Quebeck Task Force, as an acceleration-deceleration mechanism of energy transfer to the cervical spine, commonly resulting in a variety of clinical manifestations (whiplash-associated disorders) [24]. Participants were eligible if they reported neck pain following a motor vehicle crash, consistent with a whiplash injury of grade I-III, [24] were at least 17 years of age, and were within 28 days of injury. Participants were excluded if they had a pre-existing cognitive impairment affecting ability to consent, or had suffered severe physical injury (e.g. fracture (WAD IV), spinal cord injury) or psychological trauma (e.g. death of a family member). Complete inclusion and exclusion criteria are described elsewhere [25].

Primary HCPs were included if they were a primary care practitioner treating a participant with whiplash, were contactable, and provided informed consent.

\section{Setting}

Participants with whiplash were recruited from public hospital emergency departments, private physiotherapy practices and State Insurance Regulatory Authority (SIRA) databases in NSW, Australia. The SIRA databases include the Personal Injury Register (PIR), where it is mandatory for insurance claims to be reported in NSW, and the Claims Advisory Service (CAS) database, which provides support for the assessment and resolution of motor vehicle accident compensation claims.

Participants with whiplash were contacted by telephone by the research team. Eligibility was established and informed consent obtained. Participants with whiplash completed baseline and 3-month follow-up questionnaires. At 3 months, participants were asked to identify their primary $\mathrm{HCP}$, defined as their main primary health care provider following injury. This study was approved by the Sydney Local Health District Ethics Committee; reference number HREC/13/CRGH/67.

\section{Baseline questionnaires}

Baseline questionnaires assessed demographic information, pain and disability, risk status, psychological factors and general health. Demographic data captured included age, gender, weight, height, martial status, education level and country of birth.

Pain was assessed at baseline using the NRS. The NRS is frequently used in healthcare settings, [26, 27] and ranges from $0 / 10$ (no pain) to $10 / 10$ (worst pain possible). Pain catastrophising was assessed using the pain catastrophising scale (PCS), a 13-item questionnaire with scores $\geq 25 / 52$ representing a clinically significant level of pain catastrophising [28]. Patient self-reported disability was assessed using the NDI [29]. The NDI is widely used in whiplash cohorts [30-32] and ranges from 0/50 (no disability due to neck pain) to 50/50 (maximum disability due to neck pain).

Baseline risk status was assessed using a validated CPR [18]. This CPR uses baseline NDI, age and the hyperarousal sub-scale of the Post-traumatic Diagnostic scale (PDS) to predict the likelihood of recovery at 12 months post injury. Using the CPR, participants are classified as low-, medium- or high-risk of ongoing pain and disability.

Psychological factors assessed at baseline included post-traumatic stress symptoms, negative expectations of recovery, and depression, anxiety and stress. These constructs were chosen due to their association with non-recovery after whiplash [6]. Post-traumatic stress symptoms were measured using the revised Impact of Events Scale (IES-R), [33, 34] with higher scores indicative of greater distress and predictive of increased risk of non-recovery [6]. Expectations of recovery were assessed using question 7 within the short-form Orebro Musculoskeletal Pain Questionnaire (OMPQ; range 0/10: no perceived risk of pain becoming persistent to $10 / 10$ very large perceived risk of pain becoming persistent) [35]. The Depression, anxiety and stress scale (DASS) [36] was used to evaluate these constructs, with depression associated with poor outcomes after whiplash [6].

General health questionnaires assessed at baseline included the Short Form-12 (SF-12; version 1) Health Survey and the European Quality of Life -5 Dimensions - 3 levels (EQ5D3L) questionnaire.

\section{Three-month follow-up questionnaires}

Participants with whiplash completed three questionnaires at follow-up, assessing treatment received, recovery, and the therapeutic relationship (Additional file 1). The questionnaire regarding treatment received included questions on whether the Canadian $\mathrm{C}$-spine rule was applied correctly, the treatment received, and if and when referral to specialist clinicians occurred.

Recovery at 3 months was assessed using the NDI, and the Global Perceived Effect (GPE) scale. The GPE quantifies a patient's global improvement or deterioration over time, measured on an 11-point scale ranging from $-5 / 5$ (vastly worse) to $+5 / 5$ (completely recovered) [37] Therapeutic relationship was assessed using a modified version of the Working Alliance Theory of Change Inventory (WATOCI) scale [38]. Scores ranged from 9/45 (very poor therapeutic relationship) to $45 / 45$ (excellent therapeutic relationship). 
Primary HCPs completed similar questionnaires regarding treatment provided and therapeutic relationship. Practitioners were additionally asked to self-nominate the perceived risk status of their patient, as either low-, medium- or high-risk of non-recovery.

The Whiplash Evidence-based Care tool (WAD-Evidence tool) was derived for use in the study, and based on previous questionnaires used to measure adherence to Australian whiplash guidelines [8, 9]. It was modified to reflect the key recommendations identified from the 2014 edition [6]. Recommendations related to both content and process for management. Content-based recommendations included the correct application of the Canadian C-Spine Rule and the provision of recommended activating treatments. These treatments include advice and exercises. Process-based recommendations referred to risk identification and referral decisions. Scores were assigned for compliance with recommendations, with higher scores assigned if the practice was compliant with Grade A/B recommendations $[39,40]$ and negative scores if the practice was non-compliant with Grade $\mathrm{A} / \mathrm{B}$ recommendations. The scoring system is presented in detail in Table 1 . Scores were summed to give a total WAD-Evidence tool score (range -85 to 165), with higher scores reflecting greater compliance with EBC.

\section{Outcomes}

The primary outcome at 3-month follow-up was recovery measured by NDI and GPE. Recovery was defined as a score of 4 or more on the 11-point GPE, and a score of $\leq 4 / 50$ on the NDI, based on consensus decision and previous definitions $[17,37,41]$. We also investigated the proportion of individuals experiencing clinically significant improvements in neck disability (defined as mild to no disability; NDI $\leq 29 / 50$ [41]) and GPE ( $\geq 2$-point increase on 11-point scale [37]).

Additional secondary outcomes included the proportion of participants with whiplash receiving EBC via the WAD-Evidence tool, and the therapeutic relationship, according to primary HCP report. Correct identification of risk level of participants with whiplash and the appropriate decision-making processed around referral on the part of the primary HCP were also measured.

\section{Sample size}

A post hoc sample size calculation showed that the present design would provide $83 \%$ power to detect recovery at $\alpha=0.05$.

\section{Statistical analysis}

Statistical analyses were performed using SPSS v22 [42]. Baseline characteristics and 3-month variables of interest were summarised using descriptive statistics. The distribution of these data were assessed using the Shaprio-Wilk test and between-group comparisons were performed for normally-distributed data using t-tests or ANOVA. The Kruskal-Wallis non-parametric test was used where data were not normally distributed. Chi-Square analyses were used to assess associations between categorical variables of interest. The sample size (n) and standard deviation (SD) were also presented for each variable of interest. Where significant differences were found overall among multiple groups, post-hoc tests were used to identify which groups differed. For between-group comparisons using ANOVA, the LSD post hoc test was used.

A separate logistic regression model was derived for each outcome through a forward-stepwise process to assess the individual contribution of each predictor variable to the model.

Both known (e.g. CPR, expectations of recovery, baseline pain intensity) and potential (e.g. WAD-Evidence tool score, therapeutic relationship) predictors of recovery were assessed.

Predictors significantly $(p<0.05)$ associated with recovery, alone, were ranked in order of significance. Odds ratios with $95 \%$ confidence intervals, Nagelkerke $\mathrm{R}^{2}$ and $p$-values were reported. These predictors were then combined sequentially in a forward stepwise manner, retaining only those that continued to contribute significantly to the model. A final 'reduced' model, containing only those variables with significant contributions, was created for each outcome. The potential predictors of therapeutic relationship and WAD-Evidence tool score were then added to each model to assess their individual contribution to the model.

In order to determine whether the two predictor variables, in combination, had a different contribution to the model than they would in isolation, their interaction was assessed. A model was run in the form of $y=$ therapeutic relationship + WAD-Evidence tool score + therapeutic relationship"WAD-Evidence tool score. The significance of the interaction term (therapeutic relationship*WADEvidence tool score) in this model was then assessed. Mixed effects models were not suitable for use in the case of these data.

\section{Results}

A total of 228 participants with whiplash, and 53 primary HCPs, were recruited. Three-month data collection was completed for 160 participants, with 68 participants with whiplash lost to follow-up. Contact details were received for 123 primary HCPs, with 93 contactable and 53 consenting to participate (Fig. 1). Baseline characteristics and outcomes are 2 months for participants with whiplash are presented in Tables 2 and 3. 
Table 1 Whiplash Evidence Based Care Tool ("WAD-Evidence tool”). (Range: - 85 (poorest compliance) to + 165 (maximum compliance) with evidence based recommendations)

\begin{tabular}{|c|c|c|c|c|}
\hline Key recommendation & Measured by & $\begin{array}{l}\text { NHMRC Grade of } \\
\text { Recommendation: }\end{array}$ & Compliant & Non-compliant \\
\hline \multicolumn{5}{|l|}{ PART 1: Content } \\
\hline \multirow{2}{*}{$\begin{array}{l}\text { Apply the Canadian C-Spine rule to determine } \\
\text { whether an x-ray is required to confirm } \\
\text { suspected fracture or dislocation }\end{array}$} & Applied correctly & A & +5 & -5 \\
\hline & Applied incorrectly & A & & \\
\hline \multirow[t]{16}{*}{ Identify and provide evidence-based treatment } & Recommended treatments & & & \\
\hline & Advice & B & +20 & 0 \\
\hline & Exercise & B & +20 & 0 \\
\hline & Pharmacology & Consensus & +20 & 0 \\
\hline & $\begin{array}{l}\text { Treatments not routinely } \\
\text { recommended }\end{array}$ & & & \\
\hline & Manual therapy \& manipulation & C & +10 & 0 \\
\hline & Acupuncture & $\mathrm{D}$ & +10 & 0 \\
\hline & Surgery & Consensus & +10 & 0 \\
\hline & $\begin{array}{l}\text { Treatments with no evidence } \\
\text { for or against their use }\end{array}$ & & & \\
\hline & Pilates & Consensus & 0 & -5 \\
\hline & Massage & Consensus & 0 & -5 \\
\hline & Cervical pillows & Consensus & 0 & -5 \\
\hline & Electrotherapy & Consensus & 0 & -5 \\
\hline & $\begin{array}{l}\text { Treatments not routinely } \\
\text { recommended }\end{array}$ & & & \\
\hline & Injections & Consensus & 0 & -20 \\
\hline & Collar & A & 0 & -20 \\
\hline \multicolumn{5}{|l|}{ PART 2: Process } \\
\hline \multirow[t]{12}{*}{ Provide the appropriate number of treatments } & $\begin{array}{l}\text { Number of treatments by } \\
\text { risk level }\end{array}$ & & & \\
\hline & Low-risk & N/A & & \\
\hline & $0-3$ & & +20 & 0 \\
\hline & $4-12$ & & 0 & -5 \\
\hline & $13-20$ & & 0 & -10 \\
\hline & $>20$ & & 0 & -20 \\
\hline & High-risk & N/A & & \\
\hline & $0-5$ & & 0 & -5 \\
\hline & $6-16$ & & +20 & 0 \\
\hline & $17-24$ & & 0 & -5 \\
\hline & $25-32$ & & 0 & -10 \\
\hline & $>32$ & & 0 & -20 \\
\hline \multirow[t]{4}{*}{ Consider specialist referral if at-risk of non-recovery } & $\begin{array}{l}\text { Low-risk (no referral) } \\
\text { High-risk (referral) }\end{array}$ & N/A & +20 & 0 \\
\hline & $\begin{array}{l}\text { Time to referral } \\
<6 \text { weeks }\end{array}$ & N/A & +10 & 0 \\
\hline & Referred to WAD specialist & N/A & +20 & 0 \\
\hline & Referred to other specialist & N/A & +5 & 0 \\
\hline \multicolumn{3}{|l|}{ Total } & 165 & -85 \\
\hline
\end{tabular}




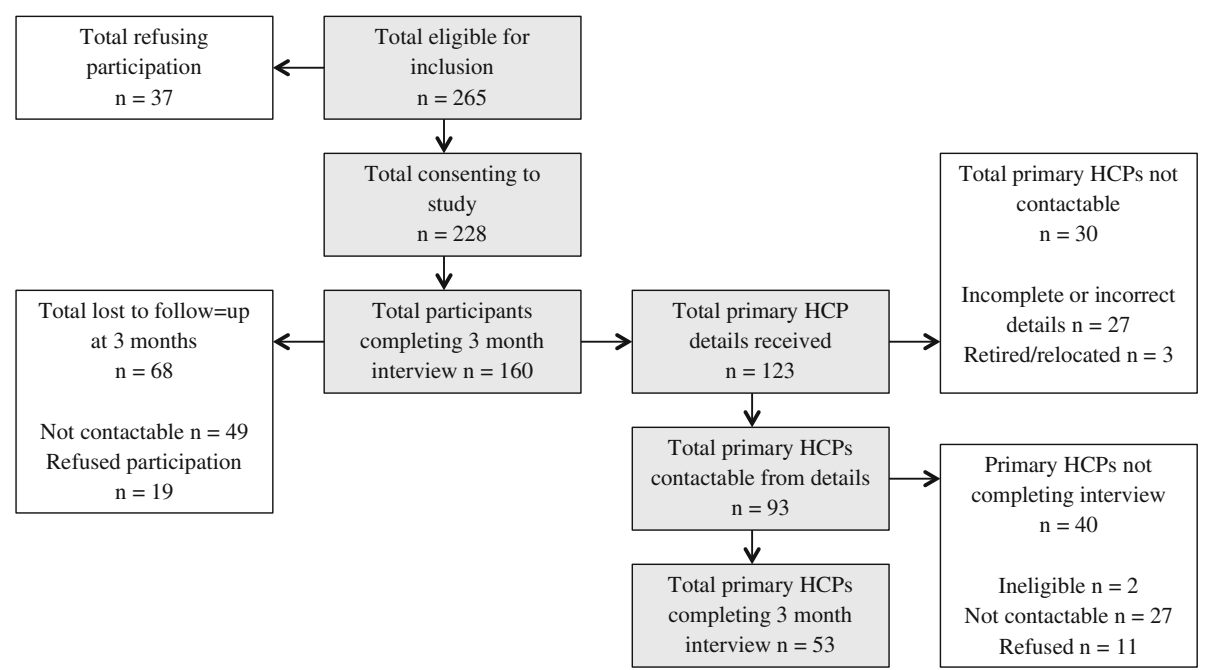

Fig. 1 Flow of participants through the study

Baseline characteristics of participants with whiplash Fifty-five percent of participants with whiplash were female, with a mean age of 42 years (Table 2). The majority (87\%) were recruited from hospital emergency departments. Four percent were recruited from physiotherapy practices, $4 \%$ from the PIR database, and 5\% from the CAS database. Correlation by centre for variables of interest was examined using correlated data models and found to be negligible (working correlation $<0.05)$.

Seventeen percent of the cohort was classified as lowrisk, $51 \%$ as medium-risk, and $32 \%$ as high-risk. Individuals in the high-risk group were higher in age $(p<0.001)$ and presented to hospital more frequently post-injury $(p=0.040)$. A significantly greater number were single $(p<0.001)$, and not currently in paid employment $(p<0.001)$. They also had a higher mean body mass index (BMI) $(p=0.038$; Table 2). Those lost-to-follow-up at 3 months had a shorter initial hospital stay, lower EQ5D3L pre injury scores and lower IES-R hyperarousal sub scale scores (e.g. mean (SD) all: 1.8(1.2), lost to follow-up: 1.5(1.1); $p=0.010$; Table 2). No other differences were observed in baseline characteristics between all participants and those lost-to-follow-up.

\section{Participants with whiplash: outcomes at 3 months}

Ninety-three percent of the cohort sought care from a primary care provider following their injury. General practitioners (GP) were most frequently consulted (84\%), followed by physiotherapists (60\%), chiropractors (13\%), massage therapists $(6 \%)$, psychologists $(3 \%)$ and osteopaths (3\%).

\section{Compliance with content messages}

According to participant self-report, the Canadian CSpine Rule was applied correctly more frequently for individuals at high-risk than for individuals at low- and medium-risk (59\% low-risk, 75\% medium-risk, 89\% high-risk; $p=0.018$; Table 3 ). Across the cohort, only $4 \%$ of participants received unnecessary imaging. However, $19 \%$ of participants interviewed reported at least one risk factor necessitating $\mathrm{x}$-ray, though had not received an $\mathrm{x}$-ray.

The majority of the cohort (>90\% across all risk groups) received recommended active treatments. A large proportion of individuals received treatments with no evidence for or against their use (e.g. massage: 52\% low-risk, 53\% medium-risk and 46\% high-risk; Fig. 2). There was no significant variation in the proportions of individuals between groups receiving treatments across all recommendation categories (Fig. 2).

\section{Compliance with process messages}

The mean number of treatments was similar between groups (mean (SD) low-risk: 8(6), medium-risk: 9(9), high-risk: $10(7) ; p=0.54)$. Only $49 \%$ of individuals at high-risk received the appropriate number of treatments (e.g. 6-16 treatments; Table 1). Similarly, the proportion of individuals that were referred for a specialist opinion across risk groups did not vary, with a very low proportion of individuals at high-risk appropriately referred (88\% low-risk, 60\% medium-risk, 36\% high-risk appropriately referred; $p<0.001$; Table 3 ). The majority of those referred were referred to traditional medical specialists including rheumatologists and neurologists (Fig. 3). 
Table 2 Baseline characteristics

\begin{tabular}{|c|c|c|c|c|c|c|c|c|}
\hline Variables & $\mathrm{N}$ & $\begin{array}{l}\text { All participants } \\
(n=215)\end{array}$ & $\begin{array}{l}\text { Lost to follow-up } \\
(n=53)\end{array}$ & $P$-value & $\begin{array}{l}\text { Low risk } \\
(n=35)\end{array}$ & $\begin{array}{l}\text { Medium risk } \\
(n=106)\end{array}$ & $\begin{array}{l}\text { High risk } \\
(n=66)\end{array}$ & $P$-value \\
\hline Age, mean (SD), in years & 205 & $42(16)$ & $41(15)$ & 0.597 & $26(6)^{* * * *}$ & $41(17)^{* * * *}$ & $53(12)^{* * * *}$ & $<0.001$ \\
\hline Gender, n (\%) & 198 & & & & & & & \\
\hline Male & & $89(45)$ & $21(44)$ & 0.848 & $12(38)$ & $43(43)$ & $32(51)$ & 0.432 \\
\hline Female & & $109(55)$ & $27(56)$ & & $20(63)$ & $56(57)$ & $31(49)$ & \\
\hline Body Mass Index, mean (SD) & 198 & $27.5(7.4)$ & $27.6(7.1)$ & 0.898 & $25.6(6.2)$ & $26.9(6.3)$ & $29.4(9.1)^{*}$ & 0.035 \\
\hline Continent of birth, $\mathrm{n}(\%)$ & 204 & & & & & & & \\
\hline Australia & & $128(60)$ & $30(63)$ & 0.952 & $26(77)$ & $65(64)$ & $32(51)$ & 0.114 \\
\hline Europe & & $19(9)$ & $5(10)$ & & $1(3)$ & $11(11)$ & $7(11)$ & \\
\hline Other & & $57(27)$ & $13(27)$ & & $7(21)$ & $26(26)$ & $24(38)$ & \\
\hline Marital status, n (\%) & 204 & & & & & & & \\
\hline Never married & & $63(29)$ & $19(40)$ & 0.089 & $21(62)$ & $34(33)$ & $8(13)$ & $<0.001$ \\
\hline Married or de facto & & $113(53)$ & $20(42)$ & & $11(32)$ & $57(55)$ & $43(68)^{*}$ & \\
\hline Divorced, widowed or separated & & $28(13)$ & $9(19)$ & & $2(6)$ & $12(12)$ & $12(19)$ & \\
\hline Position in crash, n (\%) & 204 & & & & & & & \\
\hline Driver & & $136(63)$ & $33(69)$ & 0.979 & $21(62)$ & $78(76)$ & $34(54)$ & 0.034 \\
\hline Passenger & & $32(15)$ & $7(15)$ & & $7(21)$ & $11(11)^{* *}$ & $14(22)$ & \\
\hline Motorcyclist & & $21(10)$ & $5(10)$ & & $3(9)$ & $11(11)$ & $6(10)$ & \\
\hline Bicyclist or pedestrian & & $15(7)$ & $3(6)$ & & $3(9)$ & $3(3)$ & $9(14)$ & \\
\hline Hospital presentation, mean (SD), days & 204 & $189(88)$ & $44(92)$ & 0.766 & $29(85)$ & $95(92)$ & $62(98)^{*}$ & 0.049 \\
\hline Hospital LOS, mean (SD), in days & 101 & $3.1(3.4)$ & $2.04(1.5)$ & $0.009^{*}$ & $2.07(1.3)$ & $3.1(3.6)$ & $3.6(3.7)$ & 0.412 \\
\hline Employment, mean (SD) & 204 & & & & & & & \\
\hline Paid work & & $125(58)$ & $28(58)$ & 0.328 & $26(77)$ & $68(66)$ & $28(44)$ & 0.001 \\
\hline Self-employed & & $29(14)$ & $11(23)$ & & $3(9)$ & $12(12)$ & $14(22)$ & \\
\hline Student & & $11(5)$ & $2(4)$ & & $4(12)$ & $7(7)$ & $0(0)^{*}$ & \\
\hline Retired & & $18(8)$ & $4(8)$ & & $0(0)$ & $7(7)$ & $11(18)$ & \\
\hline Other & & $21(10)$ & $3(6)$ & & $1(3)$ & $9(9)$ & $10(16)$ & \\
\hline Occupation type & 154 & & & & & & & \\
\hline Professional & & $52(24)$ & $13(33)$ & 0.829 & $13(45)$ & $22(28)$ & $16(38)$ & 0.292 \\
\hline Clerical and administrative & & $21(10)$ & $4(10)$ & & $3(10)$ & $14(18)$ & $4(10)$ & \\
\hline Technical and trades & & $18(8)$ & $3(8)$ & & $1(3)$ & $12(15)$ & $5(12)$ & \\
\hline Manager & & $22(10)$ & $7(18)$ & & $2(7)$ & $13(17)$ & $6(14)$ & \\
\hline Community and personal services & & $19(9)$ & $5(13)$ & & $7(24)$ & $7(9)$ & $5(12)$ & \\
\hline Other & & $22(10)$ & $7(18)$ & & $3(10)$ & $12(15)$ & $6(14)$ & \\
\hline \multicolumn{9}{|l|}{ Pain/ Disability } \\
\hline NDI total score $(0-50 / 50)$ & 209 & $21(9)$ & $21(10)$ & 0.875 & $11(4)^{* * * *}$ & $20(9)^{* * * *}$ & $28(5)^{* * * *}$ & $<0.001$ \\
\hline Pain during past week (NRS: 0-10/10) & 210 & $7(2)$ & $7(2)$ & 0.618 & $6(2)$ & $6(2)$ & $8(2)^{*}$ & $<0.001$ \\
\hline PCS total score $(0-52 / 52)$ & 209 & $22(14)$ & $24(14)$ & 0.302 & $16(10)$ & $20(13)$ & $30(14)^{*}$ & $<0.001$ \\
\hline PCS- Rumination (0-16/16) & 202 & $9(5)$ & $9(5)$ & 0.393 & $6(4)$ & $7(4)$ & $11(5)^{*}$ & $<0.001$ \\
\hline PCS- Magnification (0-12/12) & 203 & $6(4)$ & $6(4)$ & 0.175 & $4(3)$ & $5(3)$ & $7(3)^{*}$ & $<0.001$ \\
\hline PCS- Helplessness (0-24/24) & 202 & $10(7)$ & $10(7)$ & 0.239 & $6(5)$ & $8(7)$ & $12(6)^{*}$ & $<0.001$ \\
\hline OMPQ total score (0-210/210) & 203 & $53(18)$ & $54(19)$ & 0.500 & $46(11)$ & $49(20)$ & $65(12)^{*}$ & $<0.001$ \\
\hline OMPQ expectations of recovery $(0-10 / 10)$ & 210 & $4(3)$ & $4(3)$ & 0.899 & $3(2)$ & $4(3)$ & $6(3)^{*}$ & $<0.001$ \\
\hline
\end{tabular}


Table 2 Baseline characteristics (Continued)

\begin{tabular}{|c|c|c|c|c|c|c|c|c|}
\hline Variables & $\mathrm{N}$ & $\begin{array}{l}\text { All participants } \\
(n=215)\end{array}$ & $\begin{array}{l}\text { Lost to follow-up } \\
(n=53)\end{array}$ & $P$-value & $\begin{array}{l}\text { Low risk } \\
(n=35)\end{array}$ & $\begin{array}{l}\text { Medium risk } \\
(n=106)\end{array}$ & $\begin{array}{l}\text { High risk } \\
(n=66)\end{array}$ & $P$-value \\
\hline SF -12: MCS (0-100/100) & 209 & $43(13)$ & $43(12)$ & 0.887 & $49(13)$ & $43(13)$ & $38(11)^{*}$ & $<0.001$ \\
\hline SF -12: PhCS (0-100/100) & 209 & $33(9)$ & $33(9)$ & 0.790 & $37(9)$ & $34(10)$ & $28(6)^{*}$ & $<0.001$ \\
\hline \multicolumn{9}{|l|}{ EQ5D $3 \mathrm{~L}$} \\
\hline VAS pre-accident $(0-100 / 100)$ & 211 & $85(12)$ & $82(14)$ & $0.016^{*}$ & $86(10)$ & $85(10)$ & $84(14)$ & 0.786 \\
\hline Pre-accident (0-1/1) & 210 & $1(0.1)$ & $1(0.2)$ & 0.512 & $1(0.1)$ & $1(0.1)$ & $1(0.2)$ & 0.213 \\
\hline Post-accident (0-1/1) & 211 & $0.3(0.3)$ & $0.3(0.4)$ & 0.640 & $0.5(0.3)$ & $0.3(0.4)$ & $0.1(0.3)^{*}$ & $<0.001$ \\
\hline \multicolumn{9}{|l|}{ Psychological } \\
\hline IES-R total score (0-88/88) & 210 & $39(24)$ & $38(22)$ & 0.860 & $33(22)$ & $33(23)$ & $53(21)^{*}$ & $<0.001$ \\
\hline IES-R total sub scale score $(0-12 / 12)$ & 213 & $5.2(3.4)$ & $4.5(3.4)$ & 0.103 & $5.1(3.6)$ & $5.2(3.3)$ & $5.1(3.5)$ & 0.963 \\
\hline IES-R - Avoidance (0-4/4) & 214 & $1.7(1.2)$ & $1.6(1.1)$ & 0.521 & $1.7(1.3)$ & $1.7(1.1)$ & $1.6(1.2)$ & 0.911 \\
\hline IES-R - Intrusion (0-4/4) & 214 & $1.7(1.2)$ & $1.5(1.2)$ & 0.147 & $1.6(1.2)$ & $1.8(1.2)$ & $1.7(1.3)$ & 0.854 \\
\hline IES-R - Hyperarousal (0-4/4) & 204 & $1.8(1.2)$ & $1.5(1.1)$ & $0.010^{*}$ & $1.9(1.2)$ & $1.8(1,1)$ & $1.8(1.2)$ & 0.774 \\
\hline PDS - Hyperarousal (0-15/15) & 204 & $8(4)$ & $8(4)$ & 0.909 & $6(5)$ & $7(4)$ & $10(3)^{*}$ & $<0.001$ \\
\hline DASS total score (0-63/63) & 211 & $20(18)$ & $21(19)$ & 0.651 & $14(16)$ & $17(16)$ & $29(18)^{*}$ & $<0.001$ \\
\hline DASS - Depression (0-21/21) & 211 & $6(6)$ & $6(6)$ & 0.632 & $4(6)$ & $5(5)$ & $9(7)^{*}$ & $<0.001$ \\
\hline DASS - Anxiety (0-21/21) & 204 & $6(6)$ & $7(7)$ & 0.212 & $4(5)$ & $4(5)$ & $9(6)^{*}$ & $<0.001$ \\
\hline DASS - Stress (0-21/21) & 204 & $8(7)$ & $8(7)$ & 0.896 & $6(7)$ & $7(6)$ & $12(6)^{*}$ & $<0.001$ \\
\hline
\end{tabular}

Abbreviations: LOS Length of stay, NDI Neck Disability Index, NRS Numeric Rating Scale, PCS Pain Catastrophising Scale, OMPQ Orebro Musculoskeletal Pain Questionnaire, SF-12 Short-form 12 general health questionnaire, EQ5D 3 L Euro Quality of Life - 5 Dimensions - 3 Levels, IES-R Impact of Events Scale - revised, PTSD Post-traumatic Stress Diagnostic Scale, DASS Depression, Anxiety and Stress scale

Post hoc significance: ****low vs. med vs. high; *high vs. low and med; **med vs. low and high. $p>0.05$

WAD-Evidence tool scores ranged from -10 to 135 and did not vary between risk groups (mean (SD) low risk: 59(17), medium-risk: 60(28), high-risk: 63(32); $p=$ 0.931; Table 3), suggesting similar levels of compliance with guideline recommendations.

\section{Recovery and therapeutic relationship}

Self-reported disability due to neck pain significantly increased with risk status (mean NDI/50 (SD) low-risk: 5(7), medium-risk: 10(9), high-risk: 22(10), $p<0.001$; Table 3). Similarly the mean GPE was significantly poorer for medium- and high-risk groups compared with low risk (mean (SD) GPE range -5 to +5 : low-risk: 4(2), medium-risk: $3(2)$, high-risk: $1(2) ; p<0.001$; Table 3 ). Approximately $35 \%$ of the cohort was recovered at 3 months, with the proportion of individuals recovered reducing significantly with risk status. Fifty-eight percent of the cohort had minimal neck disability at threemonths, and $76 \%$ experienced a minimal clinically important change in perceived recovery (Fig. 4).

The therapeutic relationship was high in all groups (mean (SD) low-risk: 38 (7), medium-risk: 42 (2), highrisk 39 (7); Table 3). Seventy-five percent of participants had scores of $\geq 38 / 45$ (e.g. $85 \%$ total score), and this did not vary between risk groups (n (\%) low risk: 12 (76\%), medium-risk: 50 (82\%), high-risk: $24(67 \%) ; p=0.169)$.

\section{Primary HCPs: outcomes at 3 months}

Details were received for 123 primary HCPs, of which 93 were contactable. Of those contactable, 53 completed the 3-month questionnaire. Reasons for difficulties in contacting and obtaining questionnaires from primary HCPs are shown in Fig. 1.

\section{Compliance with content messages}

Primary HCPs reported that the Canadian C-spine rule was correctly applied to a large proportion of their patients, across all risk groups (43-75\%; Table 4). They also reported that a high proportion of their patients were provided with recommended treatments (e.g. advice $86-100 \%)$. Manual therapy and manipulation was also frequently prescribed (75-86\%; Fig. 5) across risk groups. There was no difference in the provision of care between risk groups.

\section{Compliance with process messages}

Primary HCPs reported $100 \%$ of patients at low-, and $96 \%$ of patients at medium-risk to have received appropriate referral, compared to $50 \%$ of patients at high-risk $(p<0.001$; Table 4$)$. The ability of primary HCPs to correctly identify patients at high risk of non-recovery was poor (86\% low-risk, 39\% medium-risk, 13\% high-risk correctly identified; $p=0,004$; Table 4 ). 
Table 3 Comparison of patient-reported EBC, therapeutic relationship and resource distribution between risk groups

\begin{tabular}{|c|c|c|c|c|c|c|}
\hline Variables & $\mathrm{N}$ & $\begin{array}{l}\text { All participants } \\
(n=160)\end{array}$ & $\begin{array}{l}\text { Low risk } \\
(n=24)\end{array}$ & $\begin{array}{l}\text { Medium risk } \\
(n=79)\end{array}$ & $\begin{array}{l}\text { High risk } \\
(n=48)\end{array}$ & $P$-values \\
\hline Diagnosis received, n (\%) & 159 & $112(70 \%)$ & $12(50 \%)$ & $58(74 \%)$ & $33(69 \%)$ & 0.080 \\
\hline WAD & & $59(37 \%)$ & $7(58 \%)$ & $29(50 \%)$ & $17(52 \%)$ & \\
\hline Other & & $53(31 \%)$ & $5(42 \%)$ & $29(50 \%)$ & $16(49 \%)$ & 0.871 \\
\hline \multicolumn{7}{|l|}{ Canadian Cervical Spine rule, n (\%) } \\
\hline Total x-rays required and received & 152 & $109(68 \%)$ & $13(59 \%)^{1 *}$ & $52(69 \%)^{l *}$ & $40(87 \%)$ & 0.026 \\
\hline Total x-rays required and not received & 152 & $30(19 \%)$ & $8(36 \%)^{1 *}$ & $14(19 \%)^{1 *}$ & $5(11 \%)$ & 0.042 \\
\hline Total x-rays not required and received & 152 & $6(4 \%)$ & $1(5 \%)$ & $5(7 \%)$ & $0(0 \%)$ & 0.206 \\
\hline Total x-rays not required and not received & 152 & $7(4 \%)$ & $0(0 \%)$ & $4(5 \%)$ & $1(2 \%)$ & 0.410 \\
\hline Total cases of Cervical Spine rule correctly applied & 152 & $116(73 \%)$ & $13(59 \%)$ & $56(75 \%)$ & $41(89 \%)^{*}$ & 0.018 \\
\hline \multicolumn{7}{|l|}{ Management of high - risk individuals } \\
\hline Number referred, n (\%) & 159 & $45(28 \%)$ & $3(13 \%)$ & $22(28 \%)$ & $17(36 \%)$ & 0.124 \\
\hline Time to referral, weeks, mean (SD) & 40 & $4(4)$ & $2(2)$ & $4(3)$ & $5(5)$ & 0.477 \\
\hline Referral appropriate, n (\%) & 144 & $30(19 \%)$ & $21(88 \%)^{* * *}$ & $45(60 \%)$ & $16(36 \%)$ & $<0.001$ \\
\hline Therapeutic relationship score (9-45/45), mean (SD) & 131 & $40(6)$ & $38(7)^{1 *}$ & $42(4)^{* *}$ & $39(7)$ & 0.031 \\
\hline NDI sum score (0-50/50), mean (SD) & 159 & $13(11)$ & $5(7)^{* * * *}$ & $10(9)^{* * * *}$ & $22(10)^{* * * *}$ & $<0.001$ \\
\hline GPE $(-5$ to +5$)$, mean $(S D)$ & 158 & $3(2)$ & $4(2)$ & $3(2)$ & $1(2)^{*}$ & $<0.001$ \\
\hline Resources booklet received, n (\%) & 159 & $28(17 \%)$ & $2(8 \%)$ & $12(15 \%)$ & $8(19 \%)$ & 0.512 \\
\hline Resources booklet received from, n (\%) & 29 & & & & & \\
\hline Insurer & & $6(20 \%)$ & $0(0 \%)$ & $2(15 \%)$ & $2(22 \%)$ & \\
\hline Health practitioner & & $11(40 \%)$ & $2(67 \%)$ & $3(23 \%)$ & $6(67 \%)$ & \\
\hline SIRA & & $9(31 \%)$ & $1(33 \%)$ & $6(46 \%)$ & $1(11 \%)$ & \\
\hline Other & & $3(10 \%)$ & $0(0 \%)$ & $2(15 \%)$ & $0(0 \%)$ & 0.295 \\
\hline $\begin{array}{l}\text { WAD-Evidence tool score } \\
\text { (range - 85-165), mean (SD) }\end{array}$ & 160 & $61(28)$ & $59(17)$ & $60(28)$ & $63(32)$ & 0.931 \\
\hline
\end{tabular}

Abbreviations: GPE Global perceived effect, NDI Neck Disability Index, SIRA State Insurance Regulatory Authority, WAD Whiplash associated disorder Post hoc significance: ${ }^{* * * *}$ low vs. med vs. high; ${ }^{*}$ high vs. low and med; ${ }^{* * *}$ low vs. med and high; ${ }^{*}$ low vs. medium. $p<0.05$. NB. Percentage scores for 'all participants' are calculated as a percentage of the 160 participants completing interviews

\section{Therapeutic relationship}

Therapeutic relationship was also reported to be high across all risk groups according to primary HCPs (Mean > 36/45; Table 4).

\section{Inherent bias in practitioner reporting}

It is important to consider the influence of selection and recall bias associated with the means by which practitioners were recruited to the study, and the way in which their data were collected. Practitioners had the option to participate and were asked to recall the treatments provided to their patients in retrospect. It is therefore possible that recruitment may have favoured practitioners with greater confidence in their treatment. Practitioners may have also recalled the provision of their treatment as more adherent with the guidelines than it may in fact have been.

\section{Prediction of recovery}

The univariate logistic regression analyses revealed an association between recovery and the known predictors of recovery in WAD (e.g. CPR, expectations of recovery) (Table 5). The EQ5D3L pre-injury score, and the EQ5D3L VAS score, were not significantly associated with recovery.

The first multivariate logistic regression model aimed to determine predictors of GPE. This model contained the CPR, SF-12 mental component summary (MCS) and SF-12 physical component summary (PhCS). The CPR was a good predictor of recovery within this cohort, with individuals at medium-risk of non-recovery $35 \%$ less likely to recover than those at low-risk. Individuals at high-risk of non-recovery were $80 \%$ less likely to recover than those at low-risk. The model correctly classified $75 \%$ of participants, and when therapeutic relationship was added, the model correctly classified $78 \%$ of participants. Therapeutic relationship was associated with higher odds of recovery of between 2 and 34\% (OR 1.17, 95\% CI 1.018-1.340), representing a potentially clinically significant improvement. The WAD-Evidence tool score did not contribute 


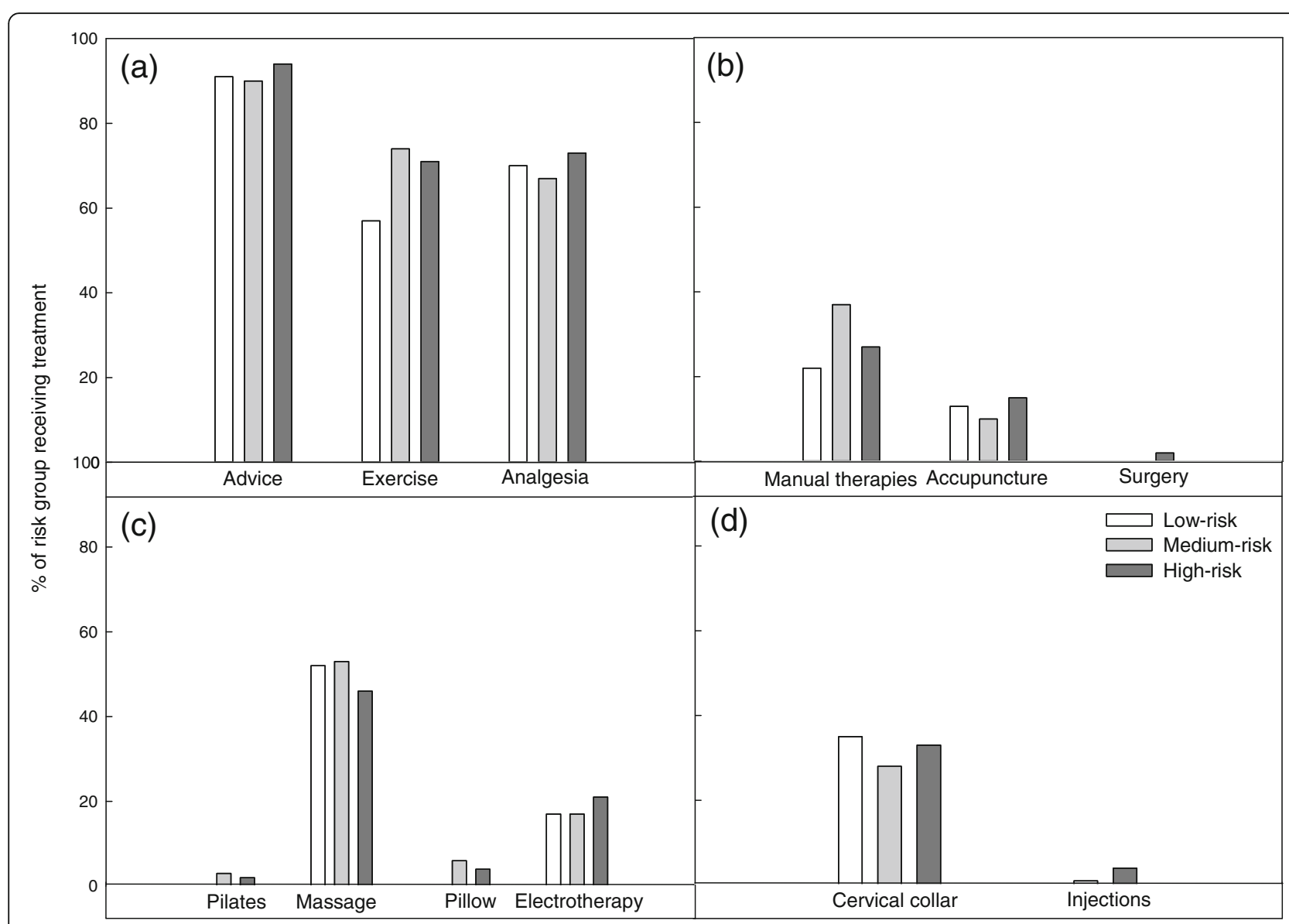

Fig. 2 Participants with whiplash: Proportion of participants with whiplash reporting treatment, stratified by risk of non-recovery. a recommended treatments, $\mathbf{b}$ treatments not routinely recommended, $\mathbf{c}$ treatments with no evidence for or against their use, $\mathbf{d}$ treatments not recommended

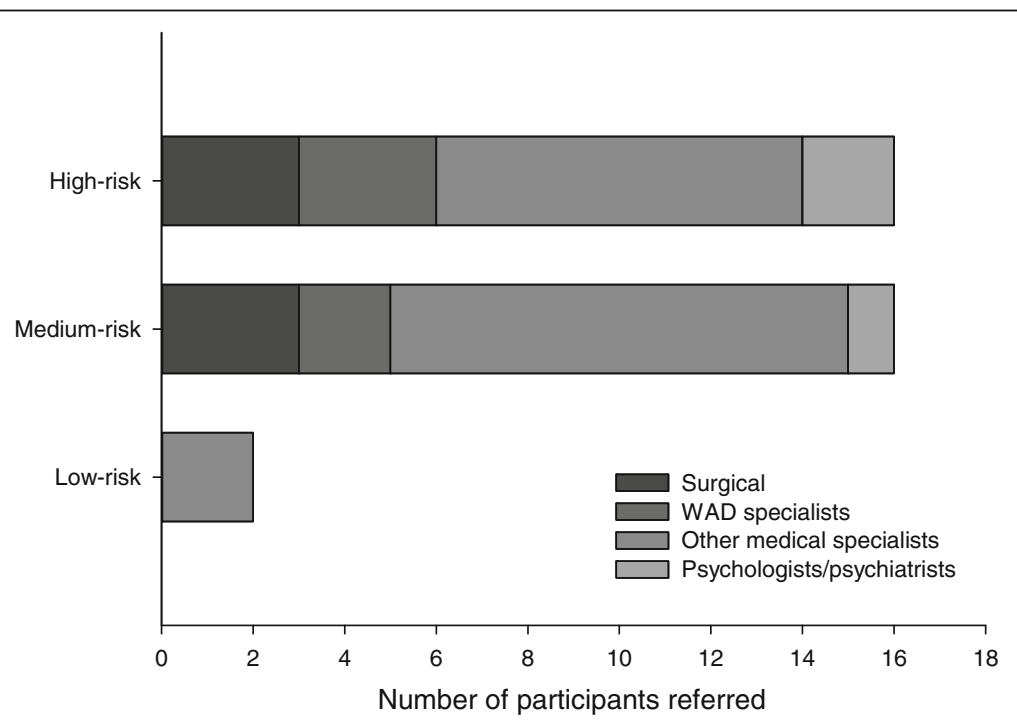

Fig. 3 Proportion of participants with whiplash referred to specialists of different disciplines at 3 months post injury 


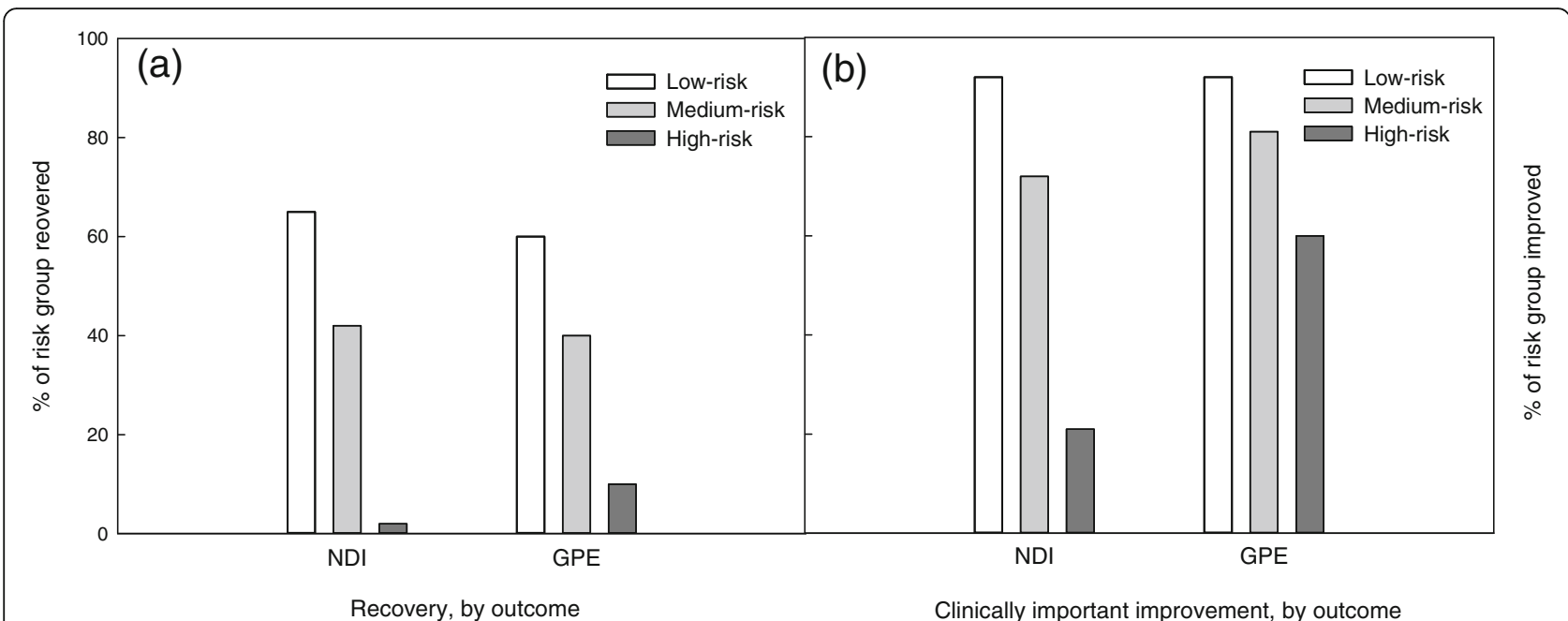

Fig. 4 Recovery and clinically important improvements at 3 months in participants with whiplash. a proportion of individuals recovered at 3 months, and $\mathbf{b}$ proportion of individuals experiencing clinically relevant improvement at 3 months, stratified by risk of non-recovery

significantly to the model (OR $0.999,95 \%$ CI $0.983-1.012$, $p=0.881$.

The second model measuring recovery via NDI scores contained the CPR, EQ5D3L post injury, and OMPQ expectations of recovery. Again, the CPR was an important predictor of recovery, with individuals at medium-risk 53\% less likely to recover than individuals at low-risk. Further, individuals at high-risk of non-recovery were $69 \%$ less likely to recover than individuals at low-risk. The model correctly classified $81 \%$ of participants. When therapeutic relationship was added to the model, it correctly classified $83 \%$ of participants. It was again associated with higher odds of recovery, between 11 and $62 \%$, and potentially clinically significant (OR 1.345, 95\% CI 1.118-1.617; Table 6). WAD-Evidence tool score did not contribute significantly to the model (OR 1.008, 95\% CI 0.990-1.026). The interaction between WAD-Evidence tool score and therapeutic relationship was also assessed, and was non-significant for both GPE $(p=0.301)$ and NDI $(p=0.905)$ outcomes.

\section{Discussion}

Evidence-based care, measured using the WAD-Evidence tool was received by, and provided to, the majority of individuals with acute whiplash in this inception cohort. Key recommendations relating to content of care and provision of recommended active treatments appeared well followed. However, guideline recommendations for a reduction in the provision of passive treatments, and recommendations for the process of care including risk identification and appropriate referral, were poorly followed. Our study also found that the CPR and the therapeutic relationship were accurate at identifying recovery at 3-months post injury. However, the provision of
EBC measured using the WAD-Evidence tool was not associated with recovery. Recommendations regarding future implementation strategies will be discussed.

The baseline characteristics of this inception cohort were typical to those of previous WAD cohorts, with the exception of gender distribution. Our cohort comprised relatively fewer females (55\%) compared to other cohorts, which are typically $60-62 \%,[31,43-46]$ though may be as high as $69 \%$, female [4]. On further investigation, gender was not found to influence recovery, for both GPE and NDI outcomes. The vast majority of our cohort were recruited from hospital emergency departments and SIRA databases, with a combined refusal rate of $32 \%$. This study was limited by the inability to compare demographics between those individuals that participated and those that declined participation, as retention of data for non-participating individuals was not possible.

The Canadian C-Spine rule was applied correctly for the majority $(74 \%)$ of this cohort, with a very low rate (4\%) of unnecessary imaging identified. This is consistent with findings from a multi-centre cluster randomised controlled trial, [47] where the rule was correctly applied for $83 \%$ of the cohort, with only $2 \%$ receiving unnecessary imaging. The trial identified a sensitivity rate of $100 \%$. However, our study revealed that a moderate proportion $(19 \%)$ of participants with whiplash did not receive an $\mathrm{x}$-ray when indicated. When the indication(s) for receiving $\mathrm{x}$-ray for these individuals were compared, the majority of our cohort (60\%) reported they were unable to turn their head at least 45 degrees bilaterally. Although difficult to determine with certainty, physicians in the hospital emergency department would likely have assessed this risk factor objectively. It is therefore 
Table 4 Comparison of primary HCP-reported evidence-based treatment provided, therapeutic relationship and resource distribution between risk groups

\begin{tabular}{|c|c|c|c|c|c|c|}
\hline Variables & $\mathrm{N}$ & $\begin{array}{l}\text { All } \\
\text { participants } \\
(n=53)\end{array}$ & $\begin{array}{l}\text { Low risk } \\
(n=7)\end{array}$ & $\begin{array}{l}\text { Medium risk } \\
(n=26)\end{array}$ & $\begin{array}{l}\text { High risk } \\
(n=16)\end{array}$ & $P$-value \\
\hline Diagnosed received, $\mathrm{n}(\%)$ & 53 & $43(81 \%)$ & $5(71 \%)$ & $22(85 \%)$ & $13(81 \%)$ & 0.725 \\
\hline WAD (grade unspecified) & & $14(35 \%)$ & $1(20 \%)$ & $6(27 \%)$ & $7(54 \%)$ & \\
\hline WAD I & & $2(5 \%)$ & $1(20 \%)$ & $1(5 \%)$ & $0(0 \%)$ & \\
\hline WAD ॥ & & $12(30 \%)$ & $2(40 \%)$ & $8(36 \%)$ & $2(15 \%)$ & \\
\hline WAD III & & $2(5 \%)$ & $0(0 \%)$ & $2(9 \%)$ & $0(0 \%)$ & \\
\hline Other & & $10(25 \%)$ & $1(20 \%)$ & $5(23 \%)$ & $4(31 \%)$ & 0.412 \\
\hline Practitioner-assessed risk level, n (\%) & 53 & & & & & \\
\hline Low & & $29(55 \%)$ & $6(86 \%)$ & $14(54 \%)$ & $8(50 \%)$ & \\
\hline Medium & & $18(34 \%)$ & $0(0 \%)$ & $10(39 \%)$ & $6(38 \%)$ & \\
\hline High & & $6(11 \%)$ & $1(14 \%)$ & $2(8 \%)$ & $2(13 \%)$ & 0.377 \\
\hline Total cases of risk assessed correctly, n (\%) & 42 & $14(26 \%)$ & $6(86 \%)^{* * *}$ & $10(39 \%)$ & $2(13 \%)$ & 0.004 \\
\hline \multicolumn{7}{|l|}{ Canadian Cervical Spine rule, n (\%) } \\
\hline Total $X$-rays required and received & 52 & $35(66 \%)$ & $3(43 \%)$ & $20(77 \%)$ & $12(75 \%)$ & 0.194 \\
\hline Total X-rays required and not received & 52 & $14(26 \%)$ & $4(57 \%)$ & $5(19 \%)$ & $3(19 \%)$ & 0.095 \\
\hline Total X-rays not required and received & 52 & $2(4 \%)$ & $0(0 \%)$ & $0(0 \%)$ & $1(6 \%)$ & 0.349 \\
\hline Total X-rays not required and not received & 52 & $1(2 \%)$ & $0(0 \%)$ & $1(4 \%)$ & $0(0 \%)$ & 0.637 \\
\hline Cases of Cervical Spine rule correctly applied, n (\%) & 52 & $36(68 \%)$ & $3(43 \%)$ & $21(80 \%)$ & $12(75 \%)$ & 0.129 \\
\hline \multicolumn{7}{|l|}{ Appropriate treatment } \\
\hline Number of treatments, mean (SD) & 53 & $12(8)$ & $6(4)^{* * *}$ & $10(8)$ & $15(9)$ & 0.035 \\
\hline Number of treatments appropriate, $\mathrm{n}(\%)$ & 43 & $14(26 \%)$ & $2(33 \%)$ & $8(36 \%)$ & $11(79 \%)$ & 0.634 \\
\hline \multicolumn{7}{|l|}{ Management of high - risk individuals } \\
\hline Number referred^, n (\%) & 53 & 19 (36\%) & $0(0 \%)^{1 *}$ & $8(31 \%)$ & $9(56 \%)$ & 0.028 \\
\hline Medical & 53 & $5(9 \%)$ & $0(0 \%)$ & $3(12 \%)$ & $2(13 \%)$ & 0.626 \\
\hline Surgical & 53 & $5(9 \%)$ & $0(0 \%)$ & $2(8 \%)$ & $2(13 \%)$ & 0.597 \\
\hline Pain specialist & 53 & $6(11 \%)$ & $0(0 \%)^{1 *}$ & $1(4 \%)$ & $5(31 \%)$ & 0.018 \\
\hline Psychologist or Psychiatrist & 53 & $5(9 \%)$ & $0(0 \%)$ & $1(4 \%)$ & $3(19 \%)$ & 0.160 \\
\hline Physiotherapy specialist & 53 & $3(6 \%)$ & $0(0 \%)$ & $1(4 \%)$ & $1(6 \%)$ & 0.781 \\
\hline Other & 53 & $1(2 \%)$ & $0(0 \%)$ & $1(4 \%)$ & $0(0 \%)$ & 0.637 \\
\hline Mean time to referral, weeks, mean (SD) & 17 & $5(4)$ & - & $6(5)$ & $5(4)$ & 0.746 \\
\hline Referral appropriate, n (\%) & 47 & $38(72 \%)$ & $7(100 \%)$ & $22(96 \%)$ & $8(50 \%)^{*}$ & $<0.001$ \\
\hline Therapeutic relationship score (9-45/45), mean (SD) & 53 & $39(4)$ & $37(5)$ & $40(4)^{m *}$ & $36(8)$ & 0.046 \\
\hline Awareness of Australian whiplash guidelines, n (\%) & 53 & $40(76 \%)$ & $5(71 \%)$ & $18(69 \%)$ & $13(81 \%)$ & 0.687 \\
\hline Name of guidelines, $\mathrm{n}(\%)$ & 14 & & & & & \\
\hline Described Australian whiplash guidelines & & $23(43 \%)$ & $3(60 \%)$ & $10(52 \%)$ & $8(73 \%)$ & \\
\hline Described other & & $10(19 \%)$ & $2(40 \%)$ & $6(32 \%)$ & $2(18 \%)$ & \\
\hline Did not describe any guidelines & & $6(11 \%)$ & $0(0 \%)$ & $2(13 \%)$ & $1(9 \%)$ & 0.715 \\
\hline Guidelines informed patient management, n (\%) & 53 & $33(62 \%)$ & $5(71 \%)$ & $15(60 \%)$ & $10(63 \%)$ & 0.859 \\
\hline
\end{tabular}

Abbreviations: GPE Global perceived effect, NDI Neck Disability Index, SIRA State Insurance Regulatory Authority, WAD Whiplash associated disorder Post hoc significance: *high vs. low and med; ${ }^{* *}$ low vs. med and high; ${ }^{1 *}$ low vs. med; ${ }^{m_{*}}$ med vs. high. $p<0.05$. NB. Percentage scores for 'all participants' are calculated as a percentage of the 53 primary health care providers completing interviews. $\wedge$ Participants with whiplash may be referred to more than one specialist practitioner 


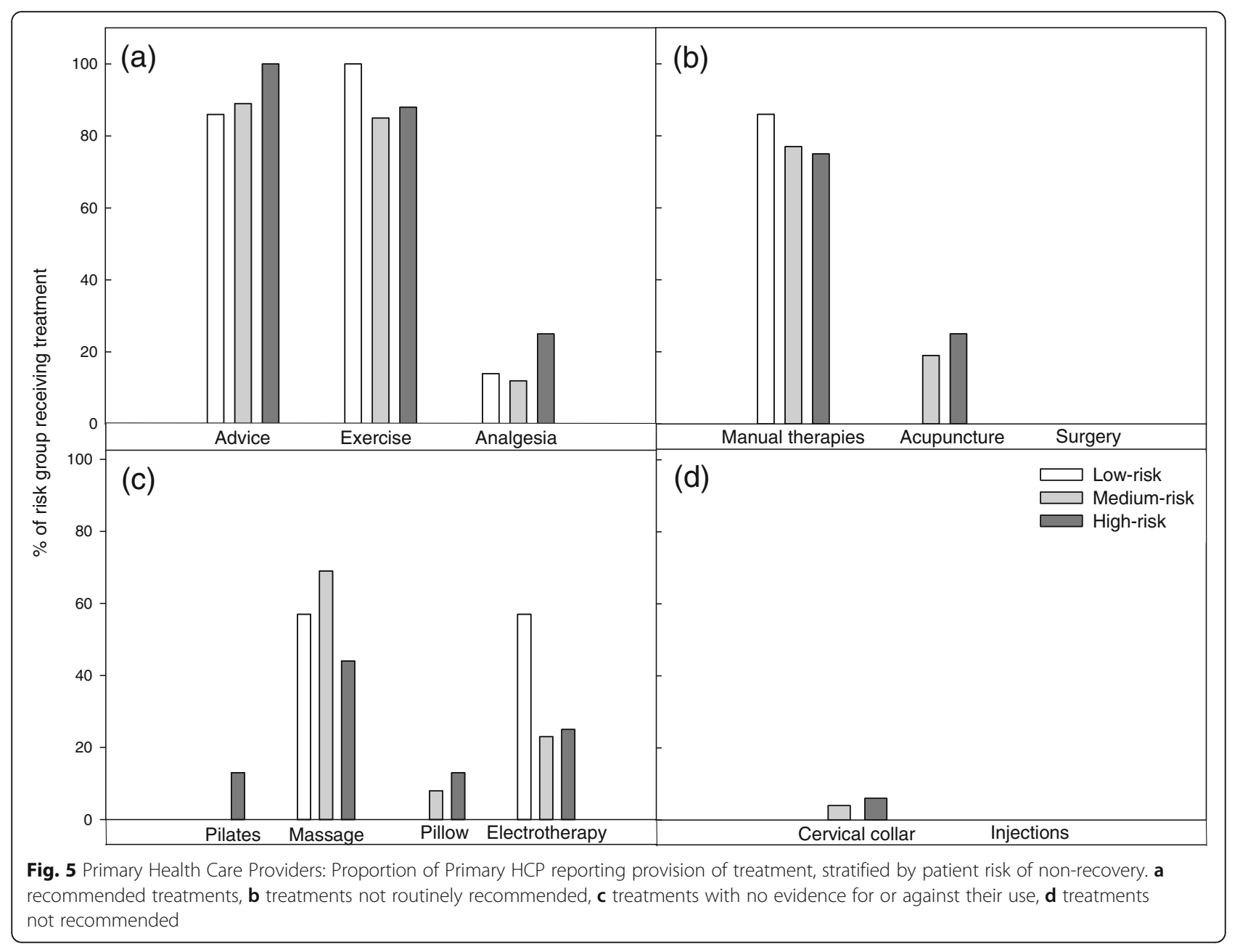

Table 5 Univariate logistic regression analysis showing relationship between previously known predictors of recovery, and recovery (GPE and NDI) at 3-months post injury

\begin{tabular}{|c|c|c|c|c|c|c|c|c|c|c|}
\hline \multirow[b]{3}{*}{ CPR (low-risk vs high-risk) } & \multicolumn{5}{|l|}{ GPE } & \multicolumn{5}{|l|}{$\mathrm{NDI}$} \\
\hline & \multirow{2}{*}{$\begin{array}{l}\text { OR } \\
13.067\end{array}$} & \multicolumn{2}{|l|}{$95 \% \mathrm{Cl}$} & \multirow{2}{*}{$\begin{array}{l}\text { Nagelkerke } \mathrm{R}^{2} \\
.198\end{array}$} & \multirow{2}{*}{$\frac{P \text {-value }}{0.001^{*}}$} & \multirow{2}{*}{$\frac{\mathrm{OR}}{82.5}$} & \multicolumn{2}{|l|}{$95 \% \mathrm{Cl}$} & \multirow{2}{*}{$\begin{array}{l}\text { Nagelkerke } \mathrm{R}^{2} \\
.337\end{array}$} & \multirow{2}{*}{$\frac{P \text {-value }}{0.000^{*}}$} \\
\hline & & 3.746 & 45.579 & & & & 9.516 & 715.277 & & \\
\hline Expectations of recovery & .768 & .669 & .881 & .147 & $<0.0001^{*}$ & .668 & .569 & .785 & .272 & $0.000^{*}$ \\
\hline Baseline pain intensity & .641 & .520 & .789 & .190 & $<0.0001^{*}$ & .630 & .510 & .778 & .201 & $0.000^{*}$ \\
\hline IES & .975 & .961 & .990 & .106 & $0.001 *$ & .967 & .952 & .983 & .174 & $0.000^{*}$ \\
\hline EQ5D3L pre & 15.843 & .748 & 335.672 & .034 & 0.076 & 10.780 & .532 & 218.251 & .026 & 0.121 \\
\hline EQ5D3L post & 9.450 & 3.196 & 27.938 & .168 & $<0.0001^{*}$ & 22.026 & 6.451 & 75.202 & .268 & $0.000^{*}$ \\
\hline EQ5D3L VAS & 1.015 & .983 & 1.048 & .008 & 0.376 & 1.018 & .985 & 1.052 & .011 & 0.283 \\
\hline SF-12 physical & 1.076 & 1.034 & 1.119 & .132 & $<0.0001^{*}$ & 1.103 & 1.055 & 1.152 & .207 & $0.000^{*}$ \\
\hline SF-12 mental & 1.078 & 1.043 & 1.114 & .212 & $<0.0001^{*}$ & 1.079 & 1.043 & 1.116 & .213 & $0.000^{*}$ \\
\hline DASS total score & .957 & .934 & .980 & .137 & $<0.0001^{*}$ & .943 & .918 & .969 & .210 & $0.000^{*}$ \\
\hline DASS depression sub scale & .894 & .834 & .958 & .107 & $0.002^{*}$ & .844 & .776 & .917 & .195 & $0.000^{*}$ \\
\hline PCS & .957 & .930 & .984 & .097 & $0.002^{*}$ & .934 & .905 & .964 & .197 & $0.000^{*}$ \\
\hline
\end{tabular}

Abbreviations: CI Confidence interval, CPR Clinical prediction rule, DASS Depression Anxiety Stress Scale, EQ5D3L Euro Quality of Life - 5 dimensions - 3 levels, IES- $R$ Impact of Events Scale - revised, OR Odds ratio, PCS Pain catastrophising scale, SF-12 Short Form Health Survey 12, VAS Visual analogue scale. * statistically significant at $p<0.05$ 
Table 6 Multivariate logistic regression predicting likelihood of recovery at 3 months post injury for GPE and NDI outcomes

\begin{tabular}{|c|c|c|c|c|c|c|}
\hline Outcome & Predictor variables & OR & $95 \% \mathrm{Cl}$ & & $p$-value & Nagelkerke $\mathrm{R}^{2}$ \\
\hline \multirow[t]{5}{*}{$\overline{\mathrm{GPE}}$} & $\begin{array}{l}\text { CPR (medium-risk vs. low-risk) } \\
\text { CPR (high-risk vs. low-risk) }\end{array}$ & $\begin{array}{l}.646 \\
.197\end{array}$ & $\begin{array}{l}.226 \\
.050\end{array}$ & $\begin{array}{l}1.849 \\
.781\end{array}$ & $\begin{array}{l}0.415 \\
0.021^{*}\end{array}$ & \\
\hline & SF-12 mental & 1.070 & 1.032 & 1.109 & $0.000^{*}$ & \\
\hline & SF-12 physical & 1.056 & 1.009 & 1.105 & $0.019^{*}$ & .364 \\
\hline & Therapeutic relationship & 1.168 & 1.018 & 1.340 & $0.026^{*}$ & .384 \\
\hline & WAD-Evidence tool score & .999 & .983 & 1.014 & 0.881 & .364 \\
\hline \multirow[t]{5}{*}{ NDla } & $\begin{array}{l}\text { CPR (medium-risk vs. low-risk) } \\
\text { CPR (high-risk vs. low-risk) }\end{array}$ & $\begin{array}{l}.462 \\
.31\end{array}$ & $\begin{array}{l}.155 \\
.003\end{array}$ & $\begin{array}{l}1.380 \\
.290\end{array}$ & $\begin{array}{l}0.167 \\
0.002^{*}\end{array}$ & \\
\hline & EQ5D3L post & 8.267 & 2.038 & 33.530 & $0.003^{*}$ & \\
\hline & OMPQ expectations & .780 & .645 & .944 & $0.011^{*}$ & .496 \\
\hline & Therapeutic relationship & 1.345 & 1.118 & 1.617 & $0.002^{*}$ & .599 \\
\hline & WAD-Evidence tool score & 1.008 & .990 & 1.026 & 0.380 & .500 \\
\hline
\end{tabular}

Abbreviations: CI Confidence interval, CPR Clinical prediction rule, EQ5D3L Euro Quality of Life - 5 dimensions - 3 levels, OR Odds ratio, OMPQ expectations OMPQ expectations of recovery, SF-12 Short Form Health Survey 12; *statistically significant at $p<0.05$

likely that the retrospective reporting of this risk factor by participants in our cohort may have increased the number of individuals testing false positive on the rule.

Provision of the guideline-recommended active treatments by primary HCPs was excellent. This suggests that the implementation of messages around the provision of these treatments have been effective. Previous implementation strategies undertaken in Australia to ensure these messages were implemented included the provision of online [7] and face-to-face $[8,9]$ education amongst insurers, [48] GP's [7] and allied health practitioners $[8,9]$. An uptake of $>80 \%$ of the activating information was reported amongst practitioners. Our data was collected 3 years after these strategies, suggesting that these messages have been retained. Therefore, a shift in focus from guideline-recommended activating treatments to those guideline recommendations less well implemented, is recommended.

A high proportion of this cohort received passive treatments that are not routinely recommended or have no evidence supporting their use, including massage (received by $50 \%$ ) and manual therapy and manipulation (received by $33 \%$ ). This recommendation was made on the basis of several trials highlighting a lack of evidence supporting the use of passive treatments including acupuncture, [49] manual therapy and manipulation, [50] and soft collars [51-53]. Our findings suggest that passive treatments are frequently applied for WAD, which is of significant concern, and supported by a recent cross-sectional survey in which $80 \%$ of Australian GPs identified manipulation as a recommended treatment [54]. Together with a reduction in the costs associated with the provision of unnecessary care, a reduction in the selection of passive treatment modalities may have benefits for the patient related to reduced dependency and improved self-efficacy.
Alternate strategies to reduce the provision of passive treatments are warranted. In other populations, guideline adherence has improved with concurrent patient and clinician education. Targeted patient education strategies, including practice-based and mailed household educational material, has reduced unnecessary antibiotic prescription for bronchitis by $40 \%$, when run concurrently with clinician education [55]. Additional strategies, including computer generated, [56] interactive [57] reminders for clinicians and audit and feedback on professional practice delivered by superiors, [58] have also improved practice, though effects were generally small.

Organisation-targeted strategies may be more effective. In a single-site before-and-after study, [59] the Canadian Cspine rule was formally adopted as emergency department policy in a community teaching hospital in Melbourne, Australia. Staff were educated on the Canadian C-Spine rule in groups and individually over a 2-month period, and provided with a reminder card, which attached to their identification badge. The result was a $25 \%$ relative reduction in X-ray ordering over a 3-month post intervention period. Similar organizational implementation strategies with the potential to reduce the provision of passive treatments may include changes to injury compensation schemes. For example, the compulsory third party (CTP) insurance compensation schemes in NSW may be revised, to exclusively support active, recommended treatments for those injured.

Organization changes may also have an important role in improving practices related to process of care. Since their inception in 1999, the Australian whiplash guidelines have recommended care be provided according to risk of non-recovery. Specifically, individuals at lower risk should receive less intervention, and individuals at medium to high risk, more. We found no difference in 
the number of treatments received by individuals in different risk groups. The implementation of the validated CPR may be the first step in improving the process of care in WAD. Given the CPR was an accurate predictor of recovery in this cohort, its use in facilitating prognosis-matched management strategies by identifying risk of non-recovery should improve recovery, and is proposed in our recent trial protocol [20]. Mandating the use of the CPR by providing insurance-generated funding for rehabilitation only if risk status is identified, and capping the number of treatments based on risk level, could aid in facilitating prognosis-matched management practices.

Individuals at high-risk of non-recovery should also receive referral to a specialist clinician with expertise in the management of WAD. Our data indicated that very few individuals are referred (36\% of individuals at highrisk), and largely to traditional medical specialists such as rheumatologists and neurologists (49\% of those referred). The lack of recommended referral practices observed in the present study could be explained by the poor ability of primary HCPs to identify individuals at high-risk of non-recovery, and poor awareness of those with expertise in whiplash [60]. Previous strategies that have improved behaviour around referral included dissemination of guidelines with structured referral sheets attached, and specialist-run clinician education programs [61-63]. It is possible that similar strategies may be effective in improving referral practices in WAD, and further investigation is warranted.

Finally, the therapeutic relationship was identified as an important predictor of recovery following WAD, yet higher scores on the WAD-evidence tool were not. The relationship between therapeutic relationship and improved outcomes identified in this cohort is consistent with previous studies in populations including low back pain, [22, 64] post-traumatic stress disorder, [65] dissociative disorders [66] and schizophrenia [67]. Of note is that this is the first study to demonstrate a relationship between therapeutic relationship and recovery following whiplash injury, and represents a significant advancement toward a better understanding of recovery after whiplash injury.

The majority of the literature suggests that patients possessing a shared sense of goals and trust with their therapist can utilise these emotions in their recovery $[66,68]$. Since the therapeutic relationship appears to have an important role in recovery, more so than the content of the care itself, the investment of time and effort on the part of the practitioner to develop and maintain a strong therapeutic relationship is warranted.

Our WAD-Evidence tool was not associated with recovery, suggesting either that receiving evidence-based care is not associated with outcome, or that our tool was not sensitive.
The known predictors of outcome in WAD are pain, disability and psychological factors [6, 17], as captured within the CPR. Since the CPR, along with the therapeutic relationship and additional outcome-specific predictor variables had already predicted a large proportion of the variance in recovery within our cohort, there is less remaining variance to explain through the addition of the WAD-Evidence tool scores. It is also important to consider that the majority of this cohort received recommended treatment, in terms of content of care. The issues identified with the provision of care related predominantly to the process of care, which may be more difficult to capture within an assessment tool.

\section{Conclusion}

Messages related to the provision of guidelinerecommended active treatments for acute WAD appear to have been successfully implemented. Therefore focus must shift to reducing the provision of passive treatments, and improving processes around the identification and appropriate management of at-risk individuals. Future work should investigate whether changes to CTP insurance funding for rehabilitation, interactive clinician education sessions, and the use of structured referral sheets disseminated within the Australian whiplash guidelines, may improve compliance.

\section{Supplementary information}

Supplementary information accompanies this paper at https://doi.org/10. 1186/s12913-019-4623-y.

Additional file 1. Questionnaire tool used to assess provision of evidence-based care.

\section{Abbreviations}

BMI: Body mass index; CAS: Claims advisory service; CPR: Clinical prediction rule; CTP: Compulsory third party; DASS: Depression, anxiety and stress scale; EBC: Evidence-based care; EQ 5D3L: European quality of life - 5 dimensions - 3 levels; GP: General practitioner; GPE: Global perceived effect; HCP: Health care practitioner; IES-R: Revised impact of events scale - revised; MCS: Mental component summary; NDI: Neck disability index; NRS: Numeric rating scale; NSW: New South Wales; OMPQ: Orebro musculoskeletal pain questionnaire; PCS: Pain catastrophising scale; PDS: Post-traumatic diagnostic scale; PhCS: Physical component summary; PIR: Personal injury register; PPV: Positive predictive value; SF-12: Short form-12; SIRA: State Insurance Regulatory Authority; VAS: Visual analogue scale; WAD: Whiplash associated disorder; WATOCI: Working Alliance Theory of Change Inventory

\section{Acknowledgements}

We are grateful to Ms. Belinda Marchant and Ms. Kathryn Higgins for their assistance in participant consent procedures and reimbursement of primary HCPs.

\section{Authors' contributions}

AG drafted the manuscript, coordinated the study, analysed and interpreted data. TR, IC, JJ, MS and JK contributed to the conception and design of the study. JJ, TR and IC drafted the funding proposal. AG and MA contributed to the acquisition of data, AK and MA contributed to the analysis, interpretation and presentation of data. All authors were involved in the revision of the manuscript and agreed on the final version of the manuscript. All authors read and approved the final manuscript. 


\section{Funding}

This study was funded by the State Insurance Regulatory Authority (SIRA) of New South Wales, Australia. The funder participated in the Steering Group for the larger FISH [23] study (Australia and New Zealand clinical trial registry identification number: ACTRN12613000889752), but had no direct involvement in the collection, analysis and interpretation of the data in this study.

IC, TR, MS and JJ are funded by National Health and Medical Research Council of Australia (NHMRC) Fellowships.

\section{Availability of data and materials}

As these results are from a larger ongoing study, which will complete followup in approximately 24 months, we will not share the data at this stage. However, the dataset will be made publicly available on completion of the larger study. The author for correspondence may be contacted regarding any specific queries on the data presented in this report.

\section{Ethics approval and consent to participate}

This study was approved by the Sydney Local Health District Ethics Committee; reference number HREC/13/CRGH/67. All participants provided informed consent prior to involvement in the study. Consent was obtained verbally, as approved in the above protocol. Consent was documented in a secure electronic datasheet by research personnel at the University of Queensland at the time of interview.

\section{Consent for publication}

N/A

\section{Competing interests}

IC is a BMC-series section editor. The authors declare there are no other competing interests.

\section{Author details}

${ }^{1}$ Faculty of Health Sciences, The University of Sydney, 75 East St, Lidcombe, NSW, Australia. ${ }^{2}$ John Walsh Centre for Rehabilitation Research, The University of Sydney, Kolling Institute, St Leonards, NSW, Australia. ${ }^{3} \mathrm{NHMRC}$ Centre of Research Excellence in Road Traffic Injury, The University of Queensland, Brisbane, QLD, Australia. ${ }^{4}$ Recover Injury Research Centre, The University of Queensland, Level 7, UQ Oral Health Centre, Herston, QLD, Australia. ${ }^{5}$ The George Institute for Global Health, The University of Sydney, Level 5, 1 King St, Newtown, NSW, Australia.

\section{Received: 8 February 2018 Accepted: 10 October 2019} Published online: 06 November 2019

\section{References}

1. Kamper SJ, Rebbeck TJ, Maher CG, McAuley JH, Sterling M. Course and prognostic factors of whiplash: a systematic review and meta-analysis. Pain. 2008;138(3):617-29.

2. Carroll LJ, Hogg-Johnson $S$, Côté $P$, et al. Course and prognostic factors for neck pain in workers. J Manip Physiol Ther. 2009;32(2):S108-S16.

3. Sterling M, Hendrikz J, Kenardy J. Compensation claim lodgement and health outcome developmental trajectories following whiplash injury: a prospective study. Pain. 2010;150(1):22-8.

4. Rebbeck T, Sindhusake D, Cameron ID, et al. A prospective cohort study of health outcomes following whiplash associated disorders in an Australian population. Inj Prev. 2006;12(2):93-8.

5. Council NHaMR. A guide to the development, implementation and evaluation of clinical practice guidelines. 1999

6. Authority SIR. Guidelines for the management of acute whiplash associated disorders for health professionals 2014. 3rd ed. Sydney: State Insurance Regulatory Authority; 2014

7. Rebbeck T, Macedo L, Paul P, Trevena L, Cameron ID. General practitioners' knowledge of whiplash guidelines improved with online education. Aust Health Rev. 2013;37(5):688-94.

8. Rebbeck T, Macedo LG, Maher CG. Compliance with clinical guidelines for whiplash improved with a targeted implementation strategy: a prospective cohort study. BMC Health Serv Res. 2013;13:213.

9. Rebbeck T, Maher CG, Refshauge KM. Evaluating two implementation strategies for whiplash guidelines in physiotherapy: a cluster-randomised trial. Aust J Physiother. 2006;52(3):165-74.
10. Grol R, Dalhuijsen J, Thomas S, Cees In 't V, Rutten G, Mokkink H. Attributes of clinical guidelines that influence use of guidelines in general practice: observational study. BMJ: Br Med J. 1998;317(7162):858-61.

11. McCormack L, Sheridan S, Lewis M, et al. Communication and dissemination strategies to facilitate the use of health-related evidence. Evid Report Technol Assess. 2013;(213):1-520. AHRQ Publication No. 13(14)-E003-EF.

12. Stiell IG, Clement CM, McKnight RD, et al. The Canadian C-spine rule versus the NEXUS low-risk criteria in patients with trauma. N Engl J Med. 2003; 349(26):2510-8.

13. Michaleff ZA, Maher C, Verhagen A, Rebbeck T, Lin CWC. Accuracy of the Canadian C-spine rule and NEXUS to screen for clinically important cervical spine injury in patients following blunt trauma: a systematic review. CMAJ. 2012;184(16):E867.

14. Mercer C, Jackson A, Moore A. Developing clinical guidelines for the physiotherapy management of whiplash associated disorder (WAD). Int J Osteopath Med. 2007;10(2):50-4.

15. Rosenfeld M, Seferiadis A, Carlsson J, et al. Active intervention in patients with whiplash-associated disorders improves long-term prognosis: a randomized controlled clinical trial. Spine. 2003;28(22):2491-8.

16. Crawford JR, Khan RJK, Varley GW. Early management and outcome following soft tissue injuries of the neck—a randomised controlled trial. Injury. 2004;35(9):891-5.

17. Ritchie C, Hendrikz J, Kenardy J, Sterling M. Derivation of a clinical prediction rule to identify both chronic moderate/severe disability and full recovery following whiplash injury. Pain. 2013;154(10):2198-206.

18. Ritchie C, Hendrikz J, Jull G, Elliott J, Sterling M. External validation of a clinical prediction rule to predict full recovery and ongoing moderate/severe disability following acute whiplash injury. J Orthop Sports Phys Ther. 2015;45(4):242-50.

19. Hill JC, Whitehurst DGT, Lewis M, et al. Comparison of stratified primary care management for low Back pain with current best practice (STarT Back): a randomised controlled trial. Lancet. 2011;378(9802):1560-71.

20. Rebbeck T, Leaver A, Bandong A, et al. Implementation of a guideline-based clinical pathway of care to improve health outcomes following whiplash injury (whiplash ImPaCT): protocol of a randomised, controlled trial. J Phys. 2016;26(6):111.

21. Horvath AO. The therapeutic alliance: concepts, research and training. Aust Psychol. 2001;36(2):170-6.

22. Ferreira PH, Ferreira ML, Maher CG, Refshauge KM, Latimer J, Adams RD. The therapeutic Alliance between clinicians and patients predicts outcome in chronic low Back pain. Phys Ther. 2013;93(4):470.

23. Priebe $\mathrm{S}, \mathrm{McC}$ abe $\mathrm{R}$. The therapeutic relationship in psychiatric settings. Acta Psychiatr Scand. 2006;113(s429):69-72.

24. Spitzer WO, Skovron ML, Salmi LR, et al. Scientific monograph of the Quebec task force on whiplash-associated disorders: redefining "whiplash" and its management. Spine. 1995;20(8 Suppl):1S.

25. Jagnoor J, Blyth F, Gabbe B, et al. Factors influencing social and health outcomes after motor vehicle crash injury: an inception cohort study protocol. BMC Public Health. 2014;14(1):199.

26. Williamson A, Hoggart B. Pain: a review of three commonly used pain rating scales. J Clin Nurs. 2005;14(7):798-804.

27. Ferreira-Valente MA, Pais-Ribeiro JL, Jensen MP. Validity of four pain intensity rating scales. Pain. 2011;152(10):2399-404.

28. Sullivan MJL, Bishop SR, Pivik J. The pain Catastrophizing scale: development and validation. Psychol Assess. 1995;7(4):524-32.

29. Vernon H, Mior $\mathrm{S}$. The neck disability index: a study of reliability and validity. J Manip Physiol Ther. 1991:14(7):409.

30. Stewart MJ, Maher CG, Refshauge KM, Herbert RD, Bogduk N, Nicholas M. Randomized controlled trial of exercise for chronic whiplash-associated disorders. Pain. 2007;128(1):59-68.

31. Sterling M, Hendriks J, Kenardy J, et al. Assessment and validation of prognostic models for poor functional recovery 12 months after whiplash injury: a multicentre inception cohort study. Pain. 2012;153(8):1727.

32. Michaleff Z, Maher C, Lin CWC, et al. Comprehensive physiotherapy exercise programme or advice for chronic whiplash (PROMISE): a pragmatic randomised controlled trial. Physiotherapy. 2015;101:e1001-e2.

33. Motlagh H. Impact of Event Scale-Revised. J Physiother. 2010;56(3):203.

34. Weiss D, Marmar C. The impact of event scale - revised. In: Wilson J, Keane T, editors. Assessing psychological trauma and PTSD. New York: Guildford Press; 1997. p. 399-411.

35. Linton SJ, Nicholas M, MacDonald S. Akademin för juridik posa, Örebro u. Development of a short form of the Örebro musculoskeletal pain screening questionnaire. Spine. 2011;36(22):1891-5. 
36. Henry JD, Crawford JR. The short-form version of the depression anxiety stress scales (DASS-21): construct validity and normative data in a large nonclinical sample. Br J Clin Psychol. 2005;44(Pt 2):227-39.

37. Kamper SJ, Ostelo RWJG, Knol DL, Maher CG, de Vet HCW, Hancock MJ. Global Perceived Effect scales provided reliable assessments of health transition in people with musculoskeletal disorders, but ratings are strongly influenced by current status. J Clin Epidemiol. 2010;63(7):760-6.e1.

38. Hall AM, Ferreira ML, Clemson L, Ferreira P, Latimer J, Maher CG. Assessment of the therapeutic alliance in physical rehabilitation: a RASCH analysis. Disabil Rehabil. 2012;34(3):257-66.

39. Guyatt GH, Oxman AD, Vist GE, et al. GRADE: an emerging consensus on rating quality of evidence and strength of recommendations. BMJ (Clinical research ed). 2008;336(7650):924-6.

40. Hillier S, Grimmer-Somers K, Merlin T, et al. FORM: an Australian method for formulating and grading recommendations in evidence-based clinical guidelines. BMC Med Res Methodol. 2011;11(1):23.

41. MacDermid J, Walton D, Avery S, Blanchard A, Etruw E, McAlpine C. Measurement properties of the neck disability index: a systematic review. JOSPT. 2009;39:400-17

42. Corp. I. IBM SPSS Statistics for Windows, Version 22.0. Armonk: IBM Corp; 2013.

43. Buitenhuis J, PJd J, JPC J, Groothoff JW. Work disability after whiplash: a prospective cohort study. SPINE. 2009;34(3):262-7.

44. Andersen TE, Karstoft Kl, Brink O, Elklit A. Pain-catastrophizing and fearavoidance beliefs as mediators between post-traumatic stress symptoms and pain following whiplash injury - a prospective cohort study. Eur J Pain. 2016;20(8):1241-52.

45. Ozegovic D, Carroll LJ, Cassidy JD. What influences positive return to work expectation? Examining associated factors in a population-based cohort of whiplash-associated disorders. Spine. 2010;35(15):E708.

46. Yang X, Côté P, Cassidy JD, Carroll L. Association between body mass index and recovery from whiplash injuries: a cohort study. Am J Epidemiol. 2007; 165(9):1063-9.

47. Stiell IG, Clement CM, Grimshaw J, et al. Implementation of the Canadian Cspine rule: prospective 12 Centre cluster randomised trial. BMJ. 2009;339:b4146.

48. Refshauge KM, Maher CG, Rebbeck TJ. Use of clinical guidelines for whiplash by insurers. Aust Health Rev. 2006;30(4):442-9.

49. Aigner N, Fialka C, Radda C, Vecsei V. Adjuvant laser acupuncture in the treatment of whiplash injuries: a prospective, randomized placebocontrolled trial. Wien Klin Wochenschr. 2006;118(3):95.

50. Teasell RW, McClure JA, Walton D, et al. A research synthesis of therapeutic interventions for whiplash-associated disorder (WAD): part 3 - interventions for subacute WAD. Pain Res Manag. 2010;15(5):305-12.

51. Borchgrevink GE, Kaasa A, McDonagh D, Stiles TC, Haraldseth O, Lereim I. Acute treatment of whiplash neck sprain injuries: a randomized trial of treatment during the first 14 days after a car accident. Spine. 1998;23(1):25-31.

52. Gennis P, Miller L, Gallagher EJ, Giglio J, Carter W, Nathanson N. The effects of soft cervical collars on persistent neck pain in patients with whiplash injury. Acad Emerg Med. 1996;3(6):568-73.

53. Teasell RW, McClure JA, Walton D, et al. A research synthesis of therapeutic interventions for whiplash-associated disorder (WAD): part 2 - interventions for acute WAD. Pain Res Manag. 2010;15(5):295-304.

54. Brijnath B, Bunzli S, Xia T, et al. General practitioners knowledge and management of whiplash associated disorders and post-traumatic stress disorder: implications for patient care. BMC Fam Pract. 2016;17:82 U6 - ctx ver=Z3988-2004\&ctx_enc=info\%3Aofi\%2Fenc\%3AUTF-8\&rfr_id=info\%3Asid\%2 Fsummonserialssolutionscom\&rft_val_fmt=info\%3Aofi\%2Ffmt\%3Akev\%3Amtx \%3Ajournal\&rftgenre= article\&rftatitle $=G e n e r a l+$ practitioners $+k$ nowledge + and + management+of+whiplash+associated+disorders+and+post-traumatic+stress + disorder\%3A+implications+for+patient+care\&rftititle $=B M C+$ family + practice \&rftau $=$ Brijnath\%2C+Bianca\&rftau $=$ Bunzli\%2C+Samantha\&rtau $=X i a \% 2 C+$ Ting\&rftau $=$ Singh\%2C+Nabita\&rftdate $=2016 \&$ rfteissn $=1471-2296 \&$ rftvolume $=$ $17 \& \mathrm{rtspage}=82 \& \mathrm{rft}$ id $=$ info\%3Apmid\%2F27440111\&rftexternalDoclD $=2744011$ 1 \&paramdict=en-US U7 - Journal Article.

55. Gonzales R, Steiner JF, Lum A, Barrett PH. Decreasing antibiotic use in ambulatory practice. JAMA. 1999;281(16):1512-9.

56. Shojania KG, Jennings A, Mayhew A, Ramsay CR, Eccles MP, Grimshaw J. The effects of on-screen, point of care computer reminders on processes and outcomes of care. Cochrane Database Syst Rev. 2009;(3):CD001096 U6 - ctx ver $=$ Z3988-2004\&ctx_enc=info\%3Aofi\%2Fenc\%3AUTF-8\&rfr_id=info\%3Asid $\% 2$ Fsummonserialssolutionscom\&rft val $\mathrm{fmt}=$ info\%3Aofi\%2Ffmt\%3Akev $\% 3 \mathrm{Amt} \times$ \%3journal\&rftgenre=article\&rftatitle $=$ The + effects + of + on-screen $\% 2 \mathrm{C}+$ point+of+care+computer+reminders+on+processes+and+outcomes+of + care\&rftititle $=$ The + Cochrane + database + of + systematic + reviews\&rftau $=$ Shoja nia\%2C+Kaveh+G\&rftau =Jennings\%2C+Alison\&rftau=Mayhew\%2C+Alain\& rftau $=$ Ramsay\%2C+Craig $+R \&$ rftdate $=2009 \&$ rfteissn $=1469-493 \times \&$ rftissue $=$ 3\&rftspage $=C D 001096 \&$ rft_id $=$ info\%3Apmid\%2F19588323\&rftexternalDocID $=$ 19588323\&paramdict=en-US U7 - Journal Article.

57. Arditi C, Rège-Walther M, Wyatt JC, Durieux P, Burnand B. Computer-generated reminders delivered on paper to healthcare professionals; effects on professional practice and health care outcomes. Cochrane Database Syst Rev. 2012;12: CD001175 U6 - ctx_ver=Z3988-2004\&ctx_enc=info\%3Aofi\%2Fenc\%3AUTF-8\&iff_id = info\%3Asid\%2Fsummonserialssolutionscom\&nft_val_fmt=info\%3Aofi\%2Ffmt\%3Akev $\% 3 A m t \times \% 3$ Ajournal\&rftgenre $=$ article\&rftatitle $=$ Computer-generated + reminders + delivered+on+paper+to+healthcare+professionals\%3B+effects+on+professionalt+ practice+and +health+care+outcomes\&rftititle $=$ The +Cochrane+database+of+ systematic+reviews\&nftau $=$ Arditi\%2C+Chantal\&rftau $=R \% C 3 \%$ A8ge-Walther $\% 2 C+$ Myriam\&nftau $=$ Wyatt\%2C+Jeremy+C\&rtau=Durieu $\% 2 \mathrm{C}+$ Pierre\& $\mathrm{rtt}$ date $=$ 2012\&rfteissn=1469-493X\&rftvolume=12\&rftspage $=C D 001175 \&$ rft_id =info\%3Apmid \%2F23235578\&rftexternalDocID=23235578\&paramdict=en-US U7 - Journal Article.

58. Ivers N, Jamtvedt G, Flottorp S, et al. Audit and feedback: effects on professional practice and healthcare outcomes. Cochrane Database Syst Rev 2012; (6): CD000259. U6 - ctx_ver=Z3988-2004\&ctx_enc=info\%3Aofi\%2 Fenc\%3AUTF-8\&rfr_id=info\%3Asid\%2Fsummonserialssolutionscom\&rft_val_ $\mathrm{fmt}=$ info\%3Aofi\%2Ffmt\%3Akev\%3Amtx\%3Ajournal\&rftgenre=article\&rftatitle= Audit+and+feedback\%3A+effects+on+professional+practice+and+healthcare+ outcomes\&rftititle $=$ The + Cochrane + database + of + systematic + reviews\&rftau $=$ Ivers\%2C+Noah\&rftau=Jamtvedt\%2C+Gro\&rftau=Flottorp\%2C+Signe\&rftau= Young\%2C + Jane + M\&rftdate $=2012 \&$ rtteissn $=1469-493 X \&$ rftissue $=6 \&$ rftspage $=$ CD000259\&rft_id=info\%3Apmid\%2F22696318\&rftexternalDoCID $=22696318 \&$ paramdict=en-US U7 - Journal Article.

59. Kerr D, Bradshaw L, Kelly A-M. Implementation of the Canadian C-spine rule reduces cervical spine $\mathrm{x}$-ray rate for alert patients with potential neck injury. J Emerg Med. 2005;28(2):127-31.

60. Bandong AN, Leaver A, Mackey M, Sterling M, Kelly J, Ritchie C, Rebbeck T. Referral to specialist physiotherapists in the management of whiplash associated disorders: perspectives of healthcare practitioners. Musculoskelet Sci Pract. 2018;34:14-26.

61. Akbari A, Mayhew A, Al-Alawi MA, et al. Interventions to improve outpatient referrals from primary care to secondary care. Cochrane Database Syst Rev. 2008:(4):CD005471 U6 - ctx ver=Z33988-2004\&ctx enc=info\%3Aofi\%2Fenc\% 3AUTF-8\&rfr_id=info\%3Asid\%2Fsummonserialssolutionscom\&rft_val_fmt=info\%3 Aofi\%2Ffmt\%3Akev\%3Amtx\%3Ajournal\&rftgenre=article\&rftatitle=Interventions+ to+improve+outpatient+referrals +from+primary+care+to+secondary+care\& rftititle $=$ The +Cochrane+database+of+systematic+reviews\&rftau $=$ Akbari\%2C+ Ayub\&rftau=Mayhew\%2C+Alain\&rftau $=$ Al-Alawi\%2C+Manal + Alawi\&rtau=Grim shaw\%2C+ Jeremy\&rftdate $=2008 \&$ rtteissn $=1469-493 X \&$ rftissue $=4 \&$ rftspage $=C D 00$ 5471\&rftid=info\%3Apmid\%2F18843691\&rftexternalDoclD=18843691\&paramdict= en-US U7 - Journal Article.

62. Bennett K, Haggard M, Churchill R, Wood S. Improving referrals for glue ear from primary care: are multiple interventions better than one alone? J Health Serv Res Policy. 2001;6(3):139-44

63. Banait $\mathrm{G}$, Sibbald $\mathrm{B}$, Thompson $\mathrm{D}$, et al. Modifying dyspepsia management in primary care: a cluster randomised controlled trial of educational outreach compared with passive guideline dissemination. Br J Gen Pract. 2003;53(487):94-100.

64. Fuentes J, Armijo-Olivo S, Funabashi M, et al. Enhanced therapeutic alliance modulates pain intensity and muscle pain sensitivity in patients with chronic low back pain: an experimental controlled study. Phys Ther. 2014;94(4):477-89.

65. Keller SM, Zoellner LA, Feeny NC. Understanding factors associated with early therapeutic alliance in PTSD treatment: adherence, childhood sexual abuse history, and social support. J Consult Clin Psychol. 2010;78(6):974.

66. Cronin E, Brand BL, Mattanah JF. The impact of the therapeutic alliance on treatment outcome in patients with dissociative disorders. Eur J Psychotraumatol. 2014,5:1-9.

67. Hewitt J, Coffey M. Therapeutic working relationships with people with schizophrenia: literature review. J Adv Nurs. 2005;52(5):561-70.

68. Elvins $\mathrm{R}$, Green J. The conceptualization and measurement of therapeutic alliance: an empirical review. Clin Psychol Rev. 2008;28(7):1167-87.

\section{Publisher's Note}

Springer Nature remains neutral with regard to jurisdictional claims in published maps and institutional affiliations. 